\title{
Decoding the molecular basis for the population mechanism of the triplet phototoxic precursors in UVA light-activated pyrimidine anticancer drugs
}

\author{
Lara Martínez-Fernández, ${ }^{[a]}$ Giovanni Granucci, ${ }^{\text {[b] }}$ Marvin Pollum,${ }^{[\mathrm{c}]}$ Carlos E. Crespo-Hernández, ${ }^{*}[\mathrm{c}]$ \\ Maurizio Persico, ${ }^{*}[\mathrm{~b}]$ Inés Corral| ${ }^{*}[\mathrm{a}]$
}

\begin{abstract}
Photosensitization of DNA by thionucleosides is a promising photo-chemotherapeutic treatment option for a variety of malignancies. DNA metabolization of thiated prodrugs can lead to cell death upon exposure to a low dose of UVA light. The exact mechanisms of thionucleoside phototoxicity are still not fully understood. In this work, we have combined femtosecond broadband transient absorption experiments with state-of-the-art molecular simulations to provide mechanistic insights for the ultrafast and efficient population of the triplet-state in the UVA-activated pyrimidine anticancer drug: 4-thiothymine. The triplet state is thought to act as a precursor to the DNA lesions and the reactive oxygen species responsible for 4-thiothymine photocytotoxicity. The electronic-structure and mechanistic results presented in this contribution reveal key molecular design criteria that can assist in developing alternative chemotherapeutic agents that may overcome some of the primary deficiencies of classical photosensitizers.
\end{abstract}

\section{Introduction}

The treatment of diseases with focused light radiation and the development of new drugs based on thio-substituted nucleic acid derivatives have both been recognized as important medical achievements within the last century. As such, each of the pioneers of these research fields have been distinguished with the Nobel Prize in Physiology or Medicine..$^{[1]}$ The synergistic effect of combining these two breakthrough advancements was not recognized until years later, when the experiments of Salet ${ }^{[2]}$, Connolly ${ }^{[3]}$, and Karran ${ }^{[4]}$, detected mutagenic damage after the cellular uptake of thio-substituted nucleobases and subsequent exposure to UVA light. In fact, these works established a new precedent by adding simple derivatives of the canonical nucleobases to the list of potent photosensitizers (PSs) for the use in photodynamic therapy (PDT) and photocrosslinking applications. PDT is used for the treatment of multiple skin conditions and a variety of superficial and internal cancers. Many PSs used in PDT work by producing singlet oxygen and other reactive species upon the absorption of light. These reactive species readily damage cellular biomolecules by causing oxidative stress or through direct reaction with substrate molecules including DNA bases, ultimately leading to cell death. Depending on whether these oxidative processes are initiated by electron transfer/hydrogen abstraction or by energy transfer between the PS and a molecular substrate, such as $\mathrm{O}_{2}$, they are

\footnotetext{
a. Universidad Autónoma de Madrid, Departamento de Química, 28049 Cantoblanco, Madrid, Spain.

b. Università di Pisa, Dipartimento di Chimica e Chimica Industriale, v. G. Moruzzi 3, 56124 Pisa, Italy.

c. Case Western Reserve University, Department of Chemistry and Center for Chemical Dynamics, 10900 Euclid Avenue, Cleveland, OH 44106, USA.
}

Supporting information for this article is given via a link at the end of the document commonly classified as Type I or Type II photosensitization mechanisms, respectively. ${ }^{[5]}$ A key difference between conventional, porphyrin-based PSs and the thionated pyrimidine and purine nucleobases is that thionucleosides are incorporated into DNA directly via nucleoside salvage pathways. ${ }^{[6]}$ The single atom replacement of an oxygen atom in the carbonyl group by a sulfur atom significantly modulates the optical properties of the nucleobases, while largely maintaining the structural integrity of the DNA. Thionation weakens the exocyclic bond(s), thus lowering the energy of the excited electronic states and making the thio-substituted nucleobases strong absorbers of UVA radiation. Conversely, the canonical nucleobases possess absorption maxima in the UVB/UVC region of the electromagnetic spectrum. ${ }^{[7]}$ Interestingly, the structural resemblance of thiobase PSs to the canonical nucleobases favors their incorporation into actively proliferating tissues, such as cancerous tissues, selectively increasing the sensitivity of these cells to UVA light. ${ }^{[8]}$ Owing to their long history as immunosuppressants, ${ }^{[9]}$ the potential use of thiopurines as photochemotherapeutics has been investigated more intensively than the thiopyrimidine counterparts. The combination of 6thioguanosine (6TG) and UVA light has been demonstrated to cause DNA damage through oxidatively-generated processes. ${ }^{[4,}$ 9a, 10] This damage has been suggested to occur primarily through Type II photoreaction mechanisms, for which the triplet excited state of 6TG has been implicated as being the precursor. ${ }^{[11]}$ Insight into the population mechanism of the triplet state of 6TG was presented at the molecular level by means of ultrafast transient absorption spectroscopy experiments, ${ }^{[12]}$ quantum-chemical calculations, ${ }^{[13]}$ and molecular dynamics simulations. ${ }^{[14]}$ Those original works reveal how thionation shapes the steeply-descending potential of the spectroscopic state of guanine, which in the canonical nucleobase is responsible for ultrafast relaxation to the ground state, while in 6 TG it results in efficient population of the triplet state and therefore substantially increases the photoreactivity.

Similar to 6TG, the 4-thiothymidine (4TT) monomer acts as a powerful Type II PS in solution, as demonstrated by its high singlet oxygen yield upon UVA excitation. ${ }^{[15]}$ However, other experimental results in which 4TT is incorporated into DNA, ${ }^{[8]}$ suggest that a photocycloaddition mechanism, leading to the formation of (6-4) photoadducts, is responsible for the observed cytotoxicity of 4TT+UVA. Recent experimental and theoretical works ${ }^{[16]}$ based on steady-state and time-resolved experiments and CASPT2 and TD-DFT calculations, have revealed ${ }^{1} \pi \pi^{*}$ excitons and charge transfer states as being the precursors of the primary mutagenic photoproducts cyclobutane thymine dimers and (6-4) adducts, respectively, in single stranded natural $(\mathrm{dT})_{20}$, whereas the contribution of triplet states in thymine dimer formation was estimated to be less than $10 \%$. Similar experimental and theoretical works on thio-substituted 
containing DNA have yet to be performed, however, the lowestenergy triplet state for these systems has been postulated as the precursor in photocrosslinking of 4-thio-substituted derivatives with the canonical pyrimidine and purine bases. ${ }^{[17]}$

In this study, we report the first comprehensive, molecular-level mechanistic analysis, based on spectroscopic and computational evidence, for populating the triplet state of 4TT. The triplet state is thought to be the precursor state responsible for the cytotoxic properties of 4TT and its effectiveness as a UVA light-activated pyrimidine anticancer drug. This joint experimental and theoretical investigation reveals a noticeably different and more complex mechanism for the population and reactivity of the long-lived triplet state in 4TT relative to 6TG. While the driving forces for generating singlet oxygen or the superoxide radical (which can lead to the formation of hydrogen peroxide) are both exergonic for 6TG, singlet oxygen formation is predicted to be less exergonic for 4TT and the formation of the superoxide radical is predicted to be endergonic, thus significantly changing the photosensitization mechanism of these two thiobases. The results presented in this work help to rationalize the different intrinsic photochemical properties observed for the thiopyrimidine versus the thiopurine prodrugs. Furthermore, our findings reveal key molecular design criteria that could be used to assist in developing alternative photochemotherapeutics that can potentially overcome some of the primary deficiencies often found in classical photosensitizers.

\section{Results and Discussion}

This contribution combines in a synergic and powerful manner steady state and broadband transient absorption spectroscopy with static and dynamic molecular modeling approaches to elucidate the mechanism leading to the population of the reactive precursor state responsible for the phototoxic properties of 4TT both in solution and in biological environments.

This section begins with an overview on the assignment of the experimental steady-state absorption and emission spectra of 4TT in different solvents. This is followed by a detailed analysis of topology of the ground- and excited-state PES in order to build a comprehensive scenario for the decay mechanism of 4TT. This static picture is further refined with a statistical analysis of the semiclassical molecular dynamics simulations, which provide a time-resolved perspective of the decay mechanism. The proposed mechanistic scaffold was then scrutinized with a sequence of experimental transient absorption spectra (TAS), resulting from the subtraction of the excited and ground state signals, at specific time delays in aqueous phosphate buffered saline (PBS) solution at $\mathrm{pH}=7.4$ and in acetonitrile, following different excitation wavelengths. Comparison of the quantum-chemically predicted TAS with those measured experimentally for 4TT allows us to present a comprehensive kinetic model that satisfactorily explains the mechanism for the population of the triplet state-the reactive state thought to be the precursor of the cytotoxic (6-4) photoadduct, ${ }^{1} \mathrm{O}_{2}$, and other reactive oxygen species (ROS). Finally, we provide an explanation for the different reactivity and singlet oxygen yields reported in the literature for the triplet state of 4TT and that of the purine-based prodrug 6TG.

Steady-state properties of 4TT. Similar to other thionated nucleobases, ${ }^{[7]}$ 4TT can be categorized as a UVA (315-400 nm) chromophore with an absorption maximum centered at ca. 335 $\mathrm{nm}$ in aqueous phosphate buffered saline (PBS) solution (Table 1). ${ }^{[15 a-c, 18]}$ The absorption spectrum is strongly red-shifted with respect to that of its parent chromophore, thymine. Thymine exhibits an absorption maximum in the UVC region at $265 \mathrm{~nm}^{[7]}$ and does not absorb UVA radiation efficiently, which would severally affect the integrity of canonical DNA. According to our multiconfigurational quantum-chemical calculations (see Methods section and Supporting Information) the lowest-energy absorption band of the 4TT chromophore (in the following 4TT is used for both 4-thiothymidine, experiments, and 4-thiothymine, calculations) originates from the overlap of two $\pi \pi^{*}$ transitions $\left(S_{2}\right.$ and $S_{3}$, Table 1$)$, which are preceded by a weakly-absorbing excited singlet state, possessing $n \pi^{*}$ character. Emission was determined to account for less than $1 \%$ of the total excited-state decay of 4TT in aqueous buffer solution (i.e., fluorescence quantum yield of $<1 \times 10^{-4}$ and phosphorescence quantum yield of $2.8 \times 10^{-4}$ in PBS). Based on a topological study of the excitedstate potential energy surfaces (PES), we have identified the $\pi \pi^{*}$ electronic states, $S_{2}$ and $T_{1}$, as being responsible for the fluorescent and phosphorescent properties of 4TT, respectively. The emission energies calculated for these states are in excellent agreement with the experimental results (See Table 1), and with previous calculated values reported for the phosphorescence. ${ }^{[15 c]}$ Interestingly, fluorescence emission originates from the $S_{2}\left(\pi \pi^{*}\right)$ state, instead of the lowest-energy singlet state, as is usually observed for most organic fluorophores.

Table 1. Experimental absorption and emission maxima and theoretical absorption and emission energies for 4TT. Calculated values in brackets correspond to the absorption values predicted by including explicit water solvent effects.

\begin{tabular}{|c|c|c|c|c|c|}
\hline \multicolumn{6}{|c|}{ Absorption } \\
\hline \multicolumn{3}{|c|}{ Experimental } & \multicolumn{3}{|c|}{ Theoretical } \\
\hline & & $\begin{array}{c}\varepsilon \\
\left(\mathrm{M}^{-1} \mathrm{~cm}^{-1}\right)\end{array}$ & & $\Delta \mathrm{E}(\mathrm{nm})$ & $f$ \\
\hline & & & $\begin{array}{l}\mathrm{S}_{0} \rightarrow \mathrm{S}_{1} \\
\mathrm{n}^{*}\end{array}$ & $\begin{array}{l}424 \\
{[419]}\end{array}$ & 0.000 \\
\hline \multirow[t]{2}{*}{$\begin{array}{l}\text { PBS } \\
\text { ACN }\end{array}$} & \multirow[t]{2}{*}{$\begin{array}{l}335 \\
337 \\
\end{array}$} & $\begin{array}{l}14000 \\
19440(16)\end{array}$ & $\begin{array}{l}\mathrm{S}_{0} \rightarrow \mathrm{S}_{2} \\
\pi \pi^{*}\end{array}$ & $\begin{array}{l}320 \\
{[326]}\end{array}$ & 0.546 \\
\hline & & & $\begin{array}{l}\mathrm{S}_{0} \rightarrow \mathrm{S}_{3} \\
\pi \pi^{*}\end{array}$ & $\begin{array}{l}287 \\
{[290]}\end{array}$ & 0.111 \\
\hline \multicolumn{6}{|c|}{ Emission } \\
\hline \multicolumn{3}{|c|}{ Experimental } & \multicolumn{3}{|c|}{ Theoretical } \\
\hline & & $\begin{array}{l}\lambda_{\max } \\
(\mathrm{nm})\end{array}$ & & \multicolumn{2}{|c|}{ (nm) } \\
\hline \multicolumn{2}{|c|}{ Fluorescence } & 400 & $\mathrm{~S}_{2} \pi \pi^{*}$ & & 30 \\
\hline \multicolumn{2}{|c|}{ Phosphorescence } & 542 & $T_{1} \pi \pi^{*}$ & & 70 \\
\hline
\end{tabular}


Photoinduced population of singlet and triplet minima.

Static picture: Figure 1 presents a schematic representation of the regions of the PES relevant to the relaxation mechanism of 4TT, which is based on the analysis of minimum energy paths. Upon excitation at $334 \mathrm{~nm}$, the $S_{2} \pi \pi^{*}$ state is populated, with no significant contributions from the $S_{3}$ or $S_{1}$ states, due to their different vertical transition energies and weaker oscillator strengths (Table 1). From the Franck-Condon region, we can identify two different pathways (PATHS I and II in Figure 1) reaching two minima in the $S_{2}$ PES that exhibit markedly different energies and geometries. One is characterized by a higher energy and a twisted conformation of the $\mathrm{C}_{5}=\mathrm{C}_{6}$ double bond (atom labelling in Figure2), denoted $\mathrm{S}_{2 \mathrm{~T}}$ (PATH I), while the other is lower in energy and has a planar conformation, denoted $S_{2 P}\left(\right.$ PATH II), (see Figure 1 inset). ${ }^{[19]}$ These two minima differ significantly, both structurally and energetically, from the CASSCF $\mathrm{S}_{2}$ minimum predicted in a previous theoretical study, ${ }^{[20]}$ and, as discussed below, are in part responsible for the photophysical properties of 4TT. The geometric and energetic differences between the planar and twisted structures could regulate the subsequent decay of the $S_{2}$ population. However, as we shall see in the discussion of the time-resolved simulation results below, the dynamics in the $S_{2}$ state do not follow Path I or Path II explicitly, rather, the trajectories tend to oscillate between planar and twisted geometries.

Our ab initio static calculations also reveal that the $\mathrm{S}_{2} / \mathrm{S}_{1}$ conical intersection $(\mathrm{Cl})$ has a twisted geometry resembling that of the $S_{2 T}$ minimum (see inset of Figure 1), and should therefore be easily accessed from it. On the contrary, a considerable degree of twisting is needed in order to access this funnel starting from the deeper planar $S_{2 P}$ minimum, which is energetically well separated from the $S_{1}$ PES and would represent a region of slower nonadiabatic decay. This may lead to the decay of the $S_{2}$ population on two different time scales, i.e., the fraction of $S_{2}$ state population visiting regions of the PES corresponding to planar ring conformations would decay on a relatively long time scale, while the rest of the population would relax to the $S_{1}$ state more rapidly. The two different time scales for the $S_{2}$ depopulation are in agreement with recent fluorescence upconversion results for $4 \mathrm{TT}^{[7,21]}$ This may lead to a significantly longer-lived singlet state $\left(\mathrm{S}_{2}\right)$ compared to other thionated nucleobases, in which this state rapidly decays to lower lying singlet and/or to triplet states. This violation of Kasha's rule ${ }^{[22]}$ can be explained on the basis of the large activation energy required to access the $S_{2} / S_{1} \mathrm{Cl}$ from the $S_{2}$ planar minimum and explains why a small amount of fluorescence from the $S_{2}$ state can be detected experimentally (Table 1$).^{[15 c]}$

The minimum of the $S_{2} / T_{2}\left(n \pi^{*}\right)$ crossing seam lies geometrically and energetically very close to the $S_{2} / S_{1} \mathrm{Cl}$, as also found in a previous work. ${ }^{[20]}$ As such, we shall indicate this region of triple quasi-degeneracy with the shorthand $S_{2} / S_{1} / T_{2} \mathrm{Cl}$. Large spinorbit couplings $\left(120 \mathrm{~cm}^{-1}\right)$ calculated at this region of the PES suggest a non-negligible probability of intersystem crossing (ISC) through this funnel, directly accessing the triplet manifold. This three-state quasi-degeneracy region also acts as a crossroad for two further relaxation pathways (PATHS A and B in Figure 1). The molecules that intersystem cross at this region of the PES and follow PATH A, populate the $T_{2}\left(n \pi^{*}\right)$ minimum and decay through the $\mathrm{T}_{2} / \mathrm{T}_{1} \quad \mathrm{Cl}$ to the most stable triplet minimum, $T_{1}\left(\pi \pi^{*}\right)$. The molecules that internally convert following PATH B, populate initially the $S_{1}\left(n \pi^{*}\right)$ minimum. The $S_{1}\left(n \pi^{*}\right)$ minimum can act as a doorway to the population of the triplet manifold, as in other nucleobase derivatives, ${ }^{[13,20]}$ due to the proximity of strongly-coupled triplet states $\left(T_{1}\right.$ and $\left.T_{2}\right)$ and to the existence of a large activation energy to reach the $\mathrm{S}_{1} / \mathrm{S}_{0} \mathrm{Cl}$ (about $2 \mathrm{eV}$, see Table S1). In this scenario, access to the triplet manifold is favored and the most stable triplet minimum, $T_{1}\left(\pi \pi^{*}\right)$, is finally populated. It should be remarked that no $T_{1} / S_{0}$ PES crossings are easily accessible from the $T_{1}\left(\pi \pi^{*}\right)$ minimum, therefore decay to the ground state is expected to be slow, leading to the trapping of a large fraction of the population in a long-lived triplet state. In a nutshell, the topology of the excited PES delineates four potentially competing paths (PATH IA, PATH IIA, PATH IB and PATH IIB) in the decay of the UVAexcited 4TT and the final trapping of the population in the lowest-energy triplet state.

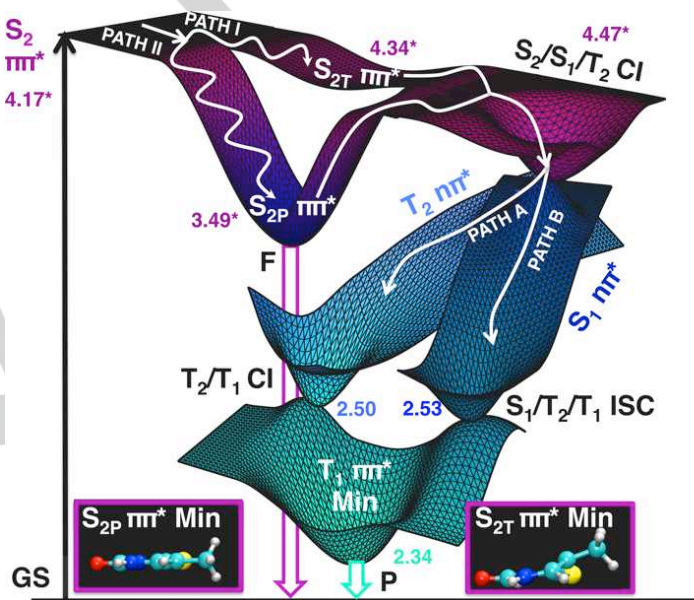

Figure 1. Schematic representation of the potential energy surfaces of 4TT relevant to the production of the precursors of cytotoxic species, based on MS-CASPT2 calculations. Relative energies to the ground state are given in $\mathrm{eV}$. Stationary points signaled with an asterisk were optimized including 7 explicit QM water molecules. In the insets are the structures of the twisted $\left(S_{2 T}\right)$ and planar $\left(S_{2 P}\right)$ minima accessed via Paths I and II, respectively. The small shift in the $S_{2}$ absorption energy with respect to Table 1 is attributed to the different number of roots considered in the two calculations.

Time resolved picture: The deactivation mechanism of excited 4TT was the object of QM/MM molecular dynamics simulations, taking into consideration the effect of a large cluster of 777 solvating molecules in the MM subsystem. Figure 2 shows the evolution of the population in the electronic states during the first 10 ps following excitation. Based on energy and transition probability criteria, our simulations were started in the spectroscopic $S_{2}\left(\pi \pi^{*}\right)$ state-consistent with its main population upon UVA excitation of 4TT. At the beginning of the simulation, all trajectories show an oscillatory motion along the twisting coordinate, which can be identified by the dihedral angle $\mathrm{N}_{1}-\mathrm{C}_{6}{ }^{-}$ $\mathrm{C}_{5}-\mathrm{C}_{7}$ (see Fig. S7-S8 in the $\mathrm{SI}$ and atom labelling in Fig. 2). This oscillation enables the molecules to explore both the planar and the twisted regions of the $S_{2}$ PES, but every hop to the $S_{1}$ state or to the triplet manifold (mainly $T_{2}$ ) occurs from a twisted conformation. Figure 2 illustrates the parallel increase of populations in both the $S_{1}$ and $T_{2}$ states, which is consistent with the continuous depopulation of the $S_{2}$ state and a continuous transfer of population from the $S_{1}$ and $T_{2}$ states to the $T_{1}$ state during this time window. 


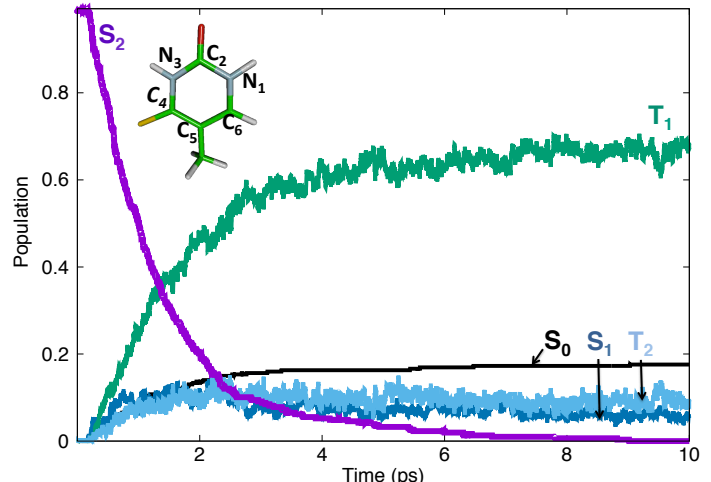

Figure 2. Time evolution of the population of different electronic states as extracted from the QM/MM dynamics simulation of $4 \mathrm{TT}$.

A quantitative analysis of the decay of the $S_{2}$ state population shows an onset time of about 200 fs during which no hops occur. This time coincides with the time needed for an oscillation of the twisting angle to reach a twisted conformation from the planar Franck-Condon geometry. Then, we see a decay best fitted by two exponentials, with time constants $\tau_{1}=1.0 \mathrm{ps}$ and $\tau_{2}=3.6 \mathrm{ps}$ and weights 0.92 and 0.08 , respectively. Fig. S7 shows that the transition rate decreases in parallel with a slight reduction in the twisting oscillation amplitude, detectable between 1 and 3 ps. The degree of vibrational excitation of the twisting coordinate may decrease in time because the most highly excited molecules decay faster and also because of internal vibrational energy redistribution and energy loss to the solvent. Independent of the pathway followed to reach the three-state $\mathrm{S}_{2} / \mathrm{S}_{1} / \mathrm{T}_{2}$ quasi-degeneracy region, our simulations suggest that the system follows preferably PATH B in Figure $1(87 \%$ of the trajectories) to further relax to the lower-energy triplet state. In fact, the molecules decaying via PATH B briefly remain in the singlet manifold, spending an average of only a few femtoseconds in the $S_{1}$ state, before reaching either $T_{2}$ or $T_{1}$ states, which are degenerate at the position of the $S_{1}$ minimum. This is consistent with the constant and low population of the $S_{1}$ state throughout the simulations (Figure 2). The remaining population (13\%) accesses the triplet manifold from the $S_{2} / S_{1} / T_{2}$ $\mathrm{Cl}$ through PATH A directly. It is worth noting that our simulations also predict that a small fraction $(16 \%)$ of the population reaching the $S_{1}$ state decays back to the ground state within $10 \mathrm{ps}$.

In summary, our dynamics simulations suggest that the primary relaxation pathway populating the triplet state in $4 \mathrm{TT}$ is $\mathrm{S}_{2 \mathrm{FC}}$ $\rightarrow \mathrm{S}_{2} / \mathrm{S}_{1} / \mathrm{T}_{2} \mathrm{Cl} \rightarrow \mathrm{S}_{1} \rightarrow \mathrm{T}_{1} \rightarrow \mathrm{T}_{1}\left(\pi \pi^{*}\right)_{\min }$ (PATH B), while the pathway $\mathrm{S}_{2 \mathrm{FC}} \rightarrow \mathrm{S}_{2} / \mathrm{S}_{1} / \mathrm{T}_{2} \quad \mathrm{Cl} \rightarrow \mathrm{T}_{2} \rightarrow \mathrm{T}_{1}\left(\pi \pi^{*}\right)_{\min }$ (PATH A) contributes to a smaller extent.

Comparison with experiment: The mechanism proposed above was experimentally scrutinized by examining the evolution of the broadband transient absorption data recorded for 4TT over 10 ps both in PBS and in ACN solutions, following excitation at $334 \mathrm{~nm}$ (Figure 3 ). The results presented herein, which were collected with an extended spectral probe window compared those previously reported in the same solvents, ${ }^{[15 b, 15 c]}$ intend to monitor the absorption signal of excited species populated along the deactivation mechanism of the initially excited 4TT. Importantly, an additional spectral feature is observed in the expanded probe region from 650 to $700 \mathrm{~nm}$. This new spectral feature can be seen most clearly in ACN solution (see Figure 3 ), thus the dynamics in PBS are provided for comparison, but much of the discussion below focuses on the transient absorption data in ACN. Upon excitation of 4TT at $334 \mathrm{~nm}$, a strong negative absorption band develops, centered at $430 \mathrm{~nm}$ in ACN, along with a positive absorption band in the 550 to $700 \mathrm{~nm}$ region, both reaching a maximum within the response time of the instrument setup (200 $\pm 50 \mathrm{fs})$. Over the first $800 \mathrm{fs}$, an excited-state absorption band grows in with an absorption maximum around $555 \mathrm{~nm}$, while the intensity of the absorption shoulder between 650 and $700 \mathrm{~nm}$ remains constant. This absorption shoulder then decays in a few picoseconds, simultaneous to a further blueshift $(50 \mathrm{~nm})$ and rise in the absorption maximum. The possibility of these newly-observed dynamics above $650 \mathrm{~nm}$ being due to vibrational cooling processes was ruled out due to its insensitivity to solvent environment, even when the non-hydrogen bonding chloroform solvent was used (see $\tau_{2}$ in Table 2). The transient absorption spectrum observed at $5 \mathrm{ps}$ matches that previously assigned to the triplet-triplet absorption band of 4TT with a long decay lifetime of $4.2 \mu \mathrm{s} .{ }^{[15 a-c, 18]}$ The experimental TAS data reveal at least two different primary relaxation pathways within the first 5 ps, which populate the long-lived triplet state of 4TT. In order to characterize these complex excited-state dynamics, vertical excitation energies were computed at regions of the excitedstate PESs predicted to be key from the static and dynamics simulations. In particular, we took the $S_{2 P}$ and $S_{2 T}$ minima as being representative of components of the wave packet (or trajectories in the semiclassical representation) visiting the planar and twisted regions of the $S_{2} P E S$, respectively, and also considered absorptions from the $S_{1}, T_{1}$, and $T_{2}$ minima. According to our calculations, we can assign the TAS signal above $650 \mathrm{~nm}$ to the planar $\mathrm{S}_{2}$ minimum and that at $600 \mathrm{~nm}$ to the twisted one, as no other species are predicted to absorb above $650 \mathrm{~nm}$ (Figure 4). In addition, our calculations show that neither of the two $S_{2}$ minima have significant absorption in the probe spectral range between 450 and $570 \mathrm{~nm}$. Similarly, the absorption spectrum of the $S_{1}$ state exhibits negligible oscillator strengths across the probed region because of its $n \pi^{*}$ character. In the same manner, the signal below $550 \mathrm{~nm}$ can be ascribed to a superposition of the absorption spectra of the $T_{1}$ and $T_{2}$ state minima. This spectral analysis demonstrates that the decay of the $S_{2}$ state population and the population of the triplet states is faster than predicted by the simulations, with a faster $\tau_{1}$ lifetime of about 200-300 fs. This discrepancy may be due to a small inaccuracy of the semiempirical PES, since the transition rate must be highly sensitive to the energy differences between the FC point, the two $S_{2}$ minima, and the $S_{2} / S_{1} / T_{2} C l$. Overall, the simulation of the experimental transient spectra using the static calculations is fully supportive of the excited-state dynamics predicted by the molecular simulations, and demonstrates the power of combining this three-pronged experimental and computational approach to elucidate complex relaxation mechanisms. 


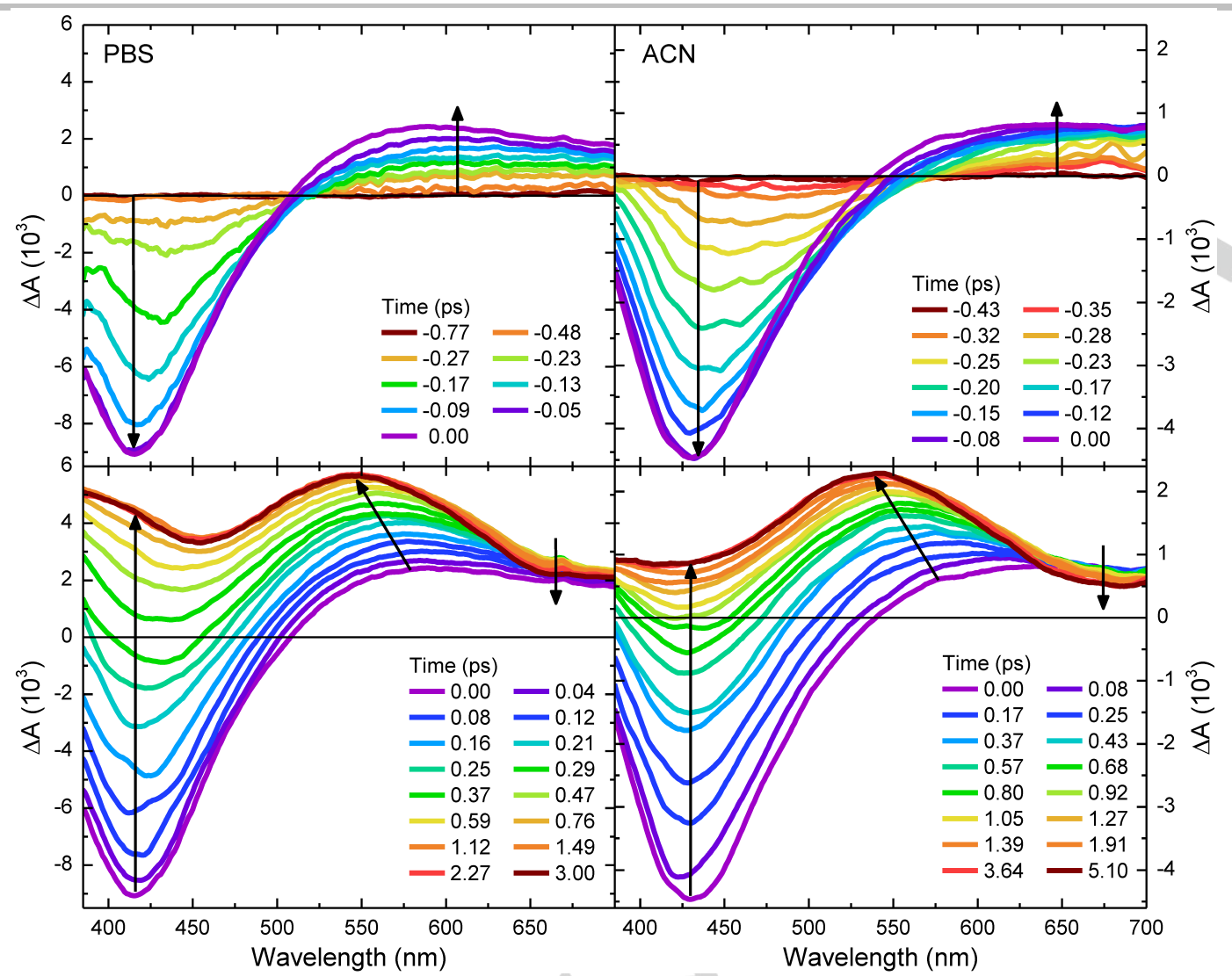

Figure 3. Transient absorption spectra collected for 4TT in PBS (left column) and acetonitrile (right column) following $334 \mathrm{~nm}$ excitation.

\section{Influence of the excitation wavelength on the triplet-state population mechanism.}

It is informative to investigate how evading the $S_{2 P}$ minimum affects the ensuing population of the triplet state because this minimum acts as a trap for a significant fraction of the $S_{2}$ population, which can decay to the ground state via photon emission according to the mechanism developed above using $334 \mathrm{~nm}$ excitation. With this goal in mind, two additional excitation wavelengths were used at 387 and $270 \mathrm{~nm}$, which were selected, respectively, to either increase the probability of exciting the $S_{1}$ state directly or to provide the system with enough electronic energy to access the $S_{3}$ state. Figure 5 provides a comparison of the key spectral differences and time delays corresponding to the maximum transient absorption signals following the three different excitation wavelengths. Representative kinetic traces and the time constants obtained from a global and target analysis of the data are presented in Figures S9-11 and Table 2, respectively.

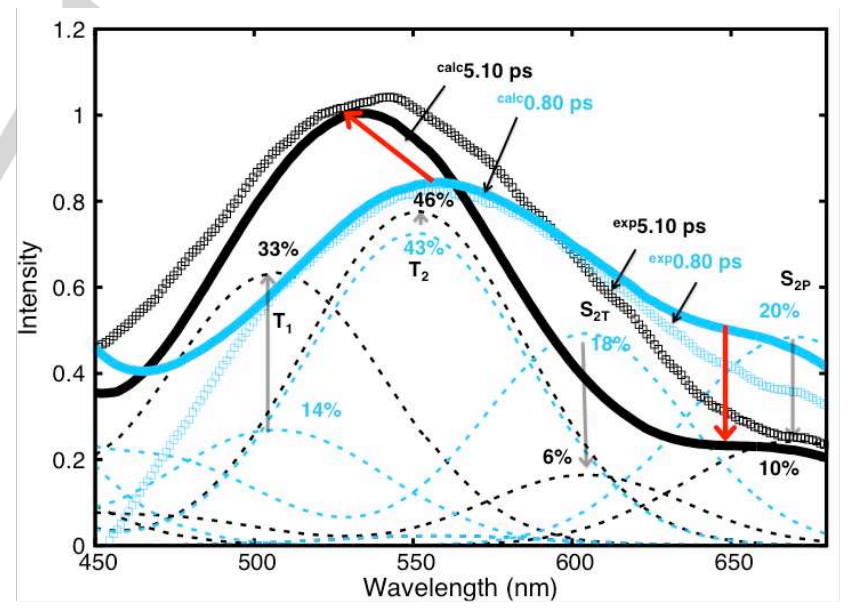

Figure 4. Comparison of the experimental and calculated transient absorption spectra at representative delay times. Remaining $S_{1}$ population $(5 \%)$ for both delay times not shown. 

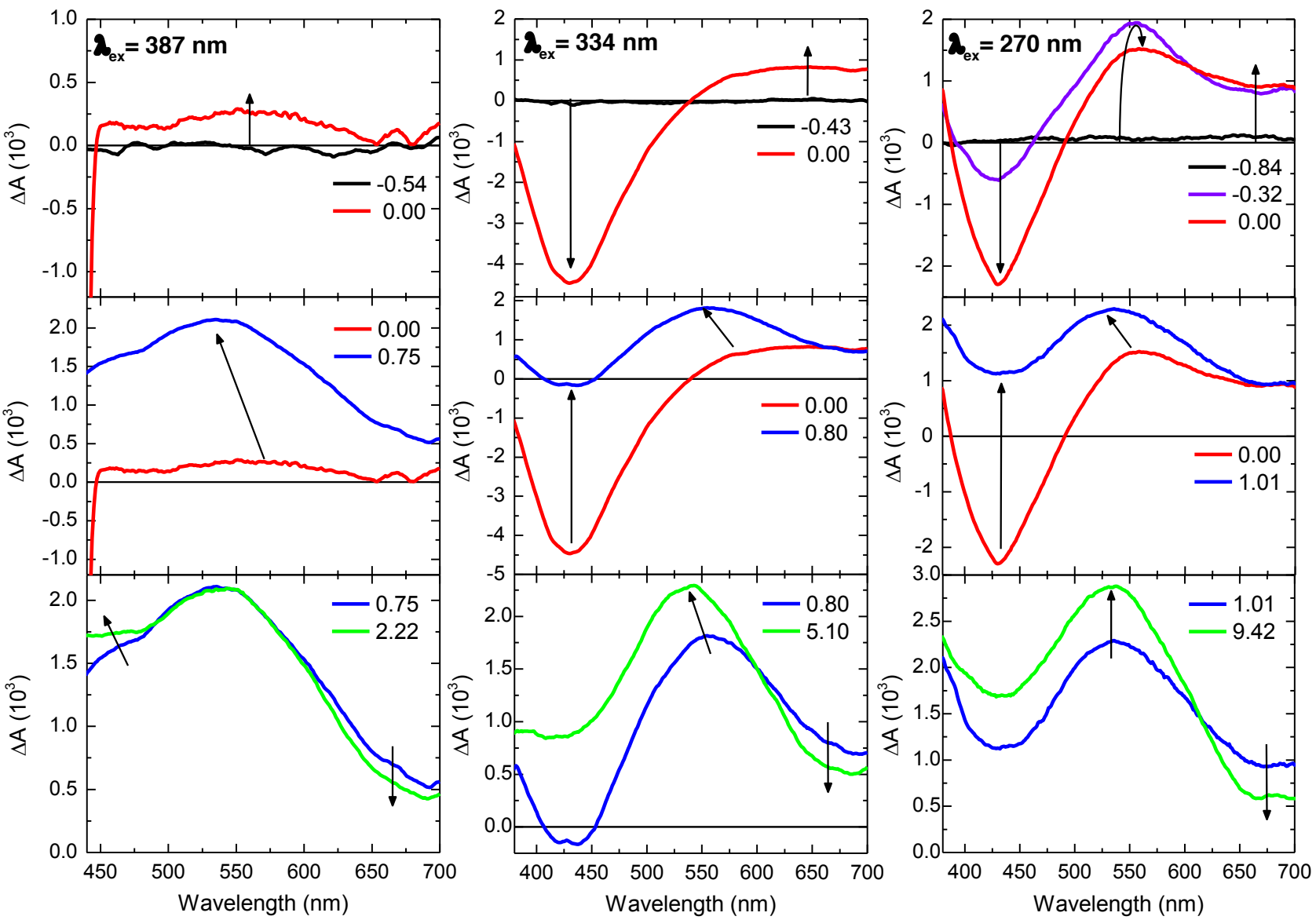

Figure 5. Transient absorption spectra of 4TT in acetonitrile following excitation at 387 (left column), 334 (middle column), and $270 \mathrm{~nm}$ (right column). For ease of comparison, spectra were selected at time delays where each excited-state signal reaches a maximum. The data following $387 \mathrm{~nm}$ excitation is cropped at 435 $\mathrm{nm}$ because the excited-state absorption is convoluted with large ground-state bleaching signals (due to the significantly higher concentration used, see Method Section for further details), thus distorting the shape of the transient spectra

As mentioned above, the experimental TAS data collected at different excitation wavelengths in Figure 5 is used to scrutinize the proposed mechanism in the previous section for the population of the reactive triplet state. Based on the ground state absorption spectrum (Table 1), an excitation wavelength of 387 $\mathrm{nm}$ is expected to primarily populate the $S_{1}$ state, from where intersystem crossing to the triplet manifold is predicted to occur on an ultrafast time scale due to the proximity of triplet states at the $S_{1}$ minimum (Figure 2). As shown in Figure 6, the rise of the triplet states takes place even faster when excitation is performed at $387 \mathrm{~nm}$ than when exciting at $334 \mathrm{~nm}$. In fact, simulation of the TAS following excitation at $387 \mathrm{~nm}$ predicts $40 \%$ of the total population to be in the triplet manifold at a time delay of $250 \mathrm{fs}$, nearly three times faster than that predicted following $334 \mathrm{~nm}$ excitation. A detailed analysis of the nonadiabatic dynamics following excitation at $387 \mathrm{~nm}$ can be found in the SI.

On the other hand, excitation of 4TT at $270 \mathrm{~nm}$ is expected to initially populate both the $S_{2}$ and $S_{3}$ states (Table 1 ), although $S_{2}$ to a larger extent. The minimum energy path starting from
Franck-Condon region of the $\mathrm{S}_{3}$ PES (Figure $\mathrm{S} 2$ of the $\mathrm{SI}$ ) shows that this state decays barrierlessly towards a $\mathrm{Cl}$ with the lower-lying $S_{2}$ state, coinciding with the position of the $S_{3}$ minimum and allowing for rapid deactivation to the $S_{2}$ state. Nevertheless, the rather flat shape of the PES at this region may favor trapping of a residual amount of population at the $S_{3}$ minimum (see supporting information, Figure S2) for long enough to be observed in our experimental measurements. In fact, the additional excited state absorption band observed at very early time delays in the experimental TAS data (Figure 5 top right panel/purple spectrum) matches very closely the calculated absorption from the $\mathrm{S}_{3}$ state minimum (Figures 7 and S6). Still, the use of higher excitation energy does not avoid the population of the $S_{2 P}$ minimum and to the contrary, it seems to increase it slightly, as deduced from the intensity of the signal above $650 \mathrm{~nm}$ in Figures 5 and 7. Hence, it can be proposed that excitation of $4 \mathrm{TT}$ with UVC $(270 \mathrm{~nm})$ radiation may actually increase the population of the $S_{2 P}$ minimum and decrease the population of the triplet state (at least to some extent) compared to excitation with UVA radiation. This excitation-wavelength dependence of the triplet yield is indeed supported 
experimentally by a comparison of the relative triplet-triplet absorption intensities, which show a small decrease with increasing excitation energy.

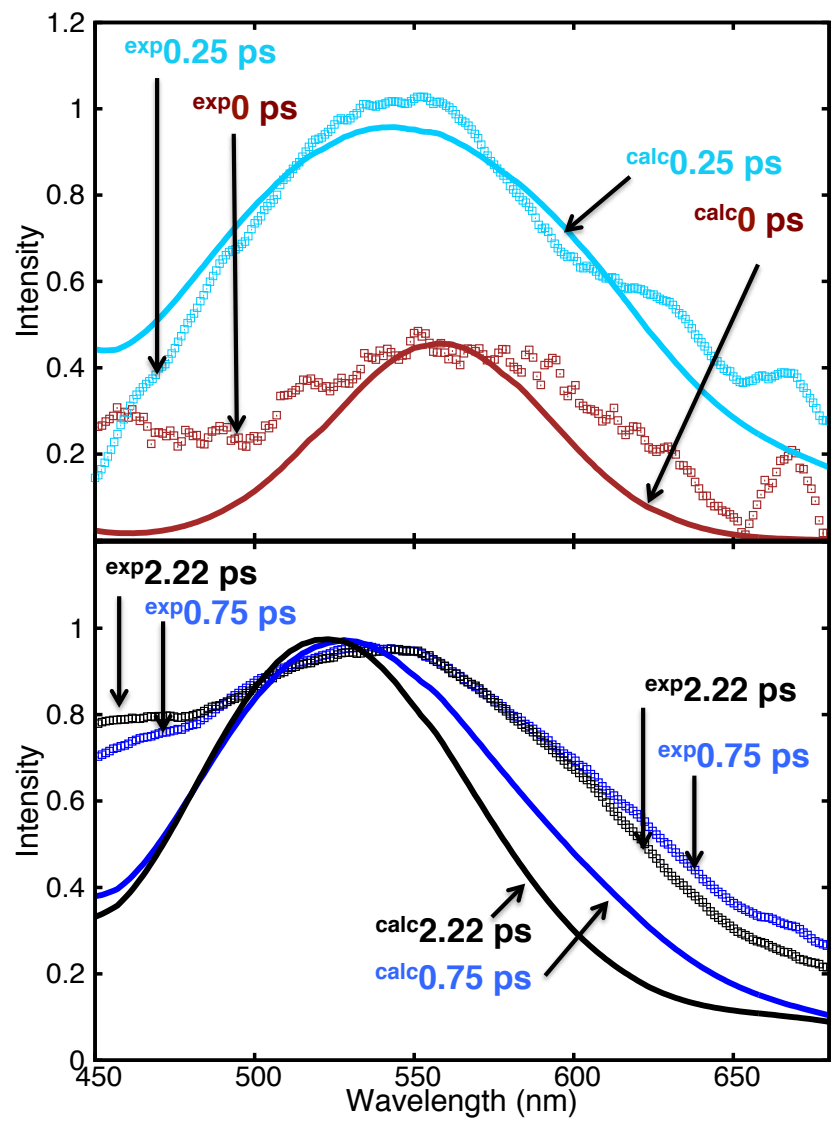

Figure 6. Comparison of the experimental and calculated transient absorption spectra in acetonitrile following excitation at $387 \mathrm{~nm}$ at different timescales (short, upper panel, and long, lower panel). The following linear combinations were used for the simulation of the transient absorption spectra: (0ps) $100 \%$ $\mathrm{S}_{1} ;(0.25 \mathrm{ps}) 20 \% \mathrm{~T}_{1}+18 \% \mathrm{~T}_{2}+45 \% \mathrm{~S}_{1}+12 \% \mathrm{~S}_{2 \mathrm{~T}}+5 \% \mathrm{~S}_{2 \mathrm{p}} ;(0.75 \mathrm{ps}) 40 \% \mathrm{~T}_{1}$ $+43 \% \mathrm{~T}_{2}+12 \% \mathrm{~S}_{2 \mathrm{~T}}+5 \% \mathrm{~S}_{2 \mathrm{P}} ;(2.22 \mathrm{ps}) 50 \% \mathrm{~T}_{1}+40 \% \mathrm{~T}_{2}+5 \% \mathrm{~S}_{2 \mathrm{~T}}+5 \% \mathrm{~S}_{2 \mathrm{P}}$

Table 2. Experimental lifetimes (in ps) in different solvents and following different excitation wavelengths (in $\mathrm{nm}$ ). Average lifetime values are reported with an approximate standard deviation of $\pm 20 \%$.

\begin{tabular}{l|l|l|l|l}
\hline Solvent & $\lambda_{\text {exc }}$ & $\tau\left(\mathrm{S}_{3}\right)$ & ISC $\left(\tau_{1}\right)$ & ISC $\left(\tau_{2}\right)$ \\
\hline ACN & 387 & - & 0.21 & 0.78 \\
\hline ACN & 334 & - & 0.54 & 1.8 \\
\hline ACN & 270 & 0.19 & 0.49 & 3.0 \\
\hline PBS & 334 & - & 0.24 & 2.1 \\
\hline PBS & 270 & 0.21 & 0.25 & 3.1 \\
\hline $\mathrm{CHCl}_{3}$ & 334 & - & 0.71 & 1.7 \\
\hline
\end{tabular}

Phototoxic properties of purine and pyrimidine thiobases: energy vs. electron transfer

The above theoretical and experimental results satisfactorily explain the population of the reactive $\mathrm{T}_{1}$ state in $4 \mathrm{TT}$, which acts as the precursor to ROS in solution and is thought to be the precursor of the (6-4) photoadduct in cells. ${ }^{[\mathrm{a}, 17]}$ However, the fate of this reactive state with molecular oxygen should also be addressed. To investigate the intrinsic reactivity of 4TT toward molecular oxygen, we have compared the likelihood of a Type I mechanism, initiated by electron transfer, (Figure 8, green arrows), versus that of a Type II, or energy transfer mechanism (Figure 8, purple arrows). These were determined based on thermodynamic arguments once the collisional complex between the excited triplet state of 4TT and the ground state of molecular oxygen has been established.

The same comparison is made for the thiopurine photosensitizer $6 \mathrm{TG}$ in order to present a rationale for the reported differences in their intrinsic phototoxic properties. It should be remarked that these predictions are strictly valid for thiobase monomers in solution, whereas additional reaction pathways are expected to compete when the thiobase is present in other biological contexts. $^{[8 a, 17]}$

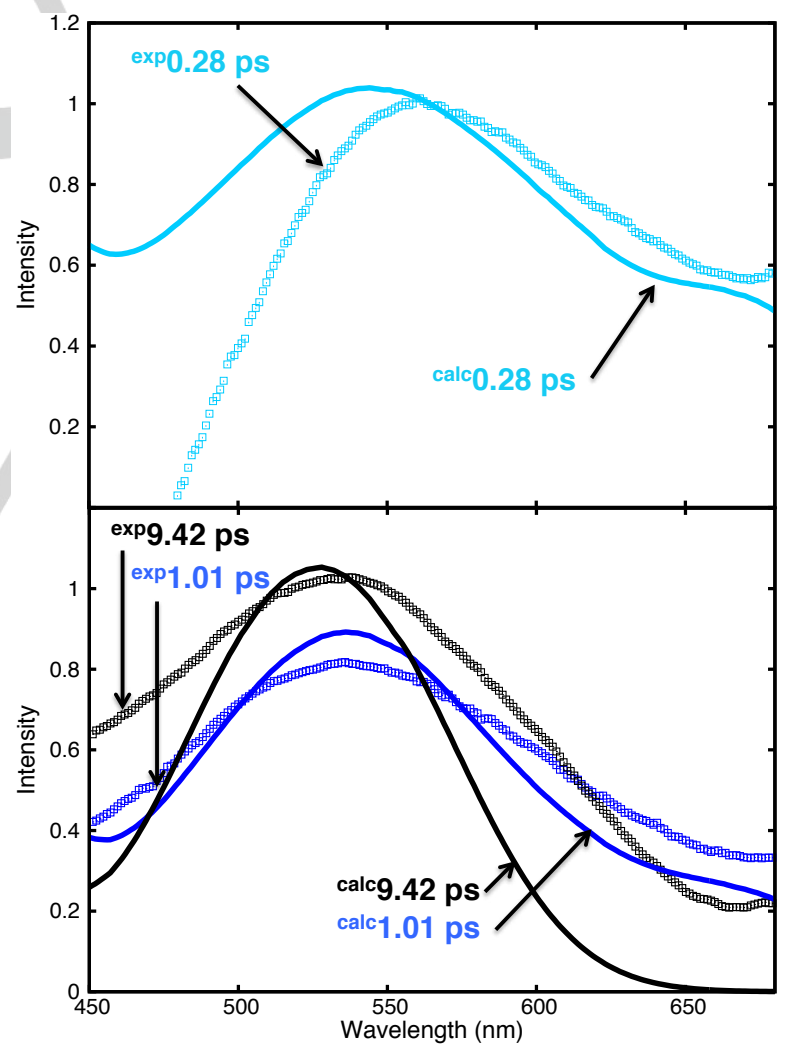

Figure 7. Comparison of the experimental and calculated transient absorption spectra in acetonitrile following excitation at $270 \mathrm{~nm}$ at different timescales (short, upper panel, and long, lower panel). The following linear combinations were used for the simulation of the transient absorption spectra: (0.28 ps) $21 \%$ $\mathrm{T}_{1}+20 \% \mathrm{~T}_{2}+4 \% \mathrm{~S}_{3}+15 \% \mathrm{~S}_{1}+20 \% \mathrm{~S}_{2 \mathrm{~T}}+20 \% \mathrm{~S}_{2 \mathrm{P}} ;(1.01 \mathrm{ps}) 30 \% \mathrm{~T}_{1}+46 \%$ $\mathrm{T}_{2}+12 \% \mathrm{~S}_{2 \mathrm{~T}}+12 \% \mathrm{~S}_{2 \mathrm{P}} ;(9.42 \mathrm{ps}) 50 \% \mathrm{~T}_{1}+50 \% \mathrm{~T}_{2}$ 
Calculations for the $\left[4 \mathrm{TT}-\mathrm{O}_{2}\right]$ complex in water solution (PCM, see $\mathrm{SI}$ ) reveal that energy transfer from the $\mathrm{T}_{1}$ minimum of 4TT to the triplet ground state of $\mathrm{O}_{2}$ is exothermic, with a change in the internal energy of the system $(\Delta \mathrm{E})$ of $-13 \mathrm{kcal} / \mathrm{mol}$. On the other hand, the electron transfer process is endothermic, with $\Delta \mathrm{E}=13 \mathrm{kcal} / \mathrm{mol}$ (see Figure 8). Importantly, both processes are exothermic for 6TG, although the triplet energy transfer process is thermodynamically preferred over the electron transfer process $(\Delta \mathrm{E}=-20 \mathrm{vs}$. $-6 \mathrm{kcal} / \mathrm{mol})$. We attribute the significantly different driving force for electron transfer in these two thiobases to their different ionization energies, the magnitude of which is larger in 4TT compared to that in 6TG (3.5 vs. $2.3 \mathrm{eV})$. The higher ionization energy hampers electron transfer from the triplet state in 4TT to the ground state of $\mathrm{O}_{2}$ and results in an endothermic reaction.

The results presented above offer a thermodynamic explanation for the noticeably different triplet-state reactivity and singlet oxygen yields reported in the literature for these two thiobases monomers under similar experimental conditions - a twofold higher singlet oxygen yield has been measured for 4TT (ca. $50 \%)^{[15 d]}$ compared to $6 \mathrm{TG}$ (ca. 25\%), ${ }^{[23]}$ even though the triplet quantum yields for both thiobases are similar. The results also suggest that the probability of forming a charge transfer complex between the triplet state of 4TT and molecular oxygen is greatly reduced, relative to the case of $6 \mathrm{TG}^{\left[{ }^{[23]}\right.}$ The exothermic nature of energy transfer from the excited triplet state of 4TT to molecular oxygen in solution may play an important role in its UVAactivated toxicity. Recent experimental results have revealed that a (6-4) photoadduct is the primary photoproduct in oligonucleotides containing 4TT exposed to UVA radiation, ${ }^{[8]}$ and no significant increase in levels of ROS were detected in 4TT treated cancer cells. This supports the idea put forward herein that the biological context in which $4 \mathrm{TT}$ is present can greatly influence the photochemistry of this PS. Hence, further work with oligonucleotides containing 4TT is needed in order to fully understand this chemistry.
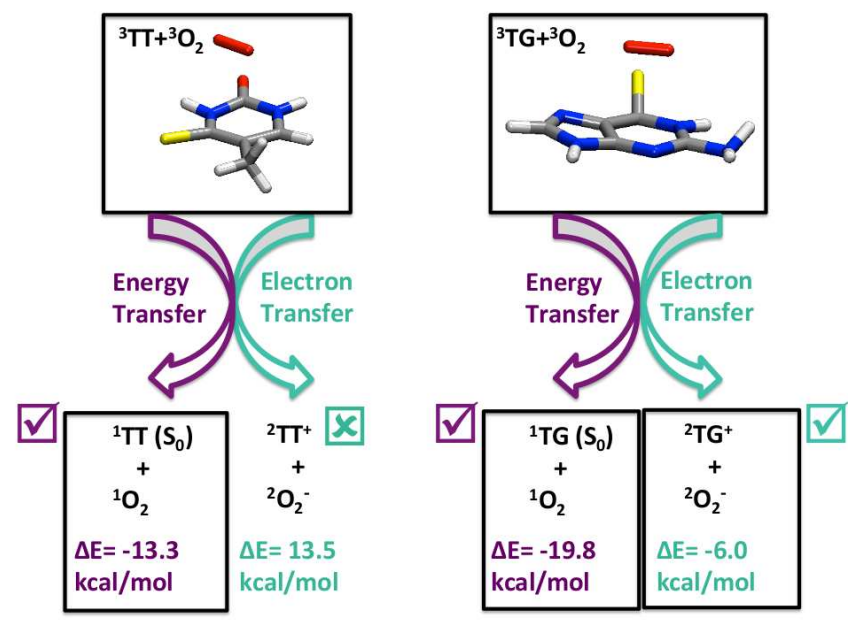

Figure 8. Thermodynamic analysis of the reactivity of triplet minima of 4TT and 6TG nucleobases towards ground state molecular oxygen $\left({ }^{3} \mathrm{O}_{2}\right)$.

\section{Conclusions}

In this study, we have successfully characterized the mechanism responsible for the population of the reactive triplet state of 4TT to an unprecedented level of molecular detail. This has been accomplished through a thorough combination of femtosecond transient absorption spectroscopy and high-level molecular simulations. The deactivation pathway leading to the long-lived triplet excited-state-the state thought to be the precursor of the cytotoxic (6-4) photoadduct and of ${ }^{1} \mathrm{O}_{2}$-is more intricate in 4TT than in other thiobase photosensitizers previously investigated. ${ }^{[13-14,20,24]}$ In fact, the existence of two $S_{2}$ minima, and of a region of triple quasi-degeneracy for the $S_{2}, S_{1}$ and $T_{2}$ states, diversifies the population dynamics in the $S_{2}$ state PES significantly, and modulates the decay of the population to the $T_{1}$ minimum. The triplet-state population dynamics are also shown to be excitation-wavelength dependent, with the triplet yield increasing as the excitation energy is decreased. In this dynamics a major role is played by the twisting of the $\mathrm{C}_{5}=\mathrm{C}_{6}$ double bond, which is necessary to reach the triple quasidegeneracy region from the FC point. The main triplet-state population mechanism appears to be $S_{2} \rightarrow S_{1} \rightarrow T_{1}$, whereas the $S_{2} \rightarrow T_{2} \rightarrow T_{1}$ pathway yields a minor contribution. A thermodynamic explanation has also been presented for the twofold larger quantum yield of singlet oxygen reported for 4TT $(50 \%)$ compared to that of the thiopurine 6TG $(25 \%)$ in solution. ${ }^{[15 \mathrm{~d}, 23]}$ The higher singlet-oxygen photosensitizing efficacy of 4 TT versus $6 T G$ is ascribed, at least partially, to the higher ionization energy of 4TT, which translates into unfavorable electron transfer reactions, enabling more efficient generation of singlet oxygen in solution.

\section{Computational and Experimental Section}

\section{Experimental methods.}

The broadband femtosecond transient absorption spectrometer has been described in detail elsewhere. ${ }^{[15 \mathrm{~d},}{ }^{25]}$ In brief, an $800 \mathrm{~nm}, 100 \mathrm{fs}$ fundamental laser source was used to generate the pump pulses of 270 , 334 , and $387 \mathrm{~nm}$ with an optical parametric amplifier and to generate the white light continuum probe pulses in a $2 \mathrm{~mm} \mathrm{CaF}_{2}$ crystal. Solutions of 4-thiothymidine were prepared in $2 \mathrm{~mm}$ cuvettes with an optical density at the pump wavelength of $1.6,1.4$ and 0.16 for the 270,334, and 387 $\mathrm{nm}$ experiments, respectively. Experiments were also performed at equal 4TT concentrations exciting at 334 or $387 \mathrm{~nm}$, and verified that the spectral differences in Figure 5 are due to the excitation wavelength and not to the concentration. All solutions were replaced before degrading more than $5 \%$. Data was collected using a homemade LabVIEW program. Global and target analysis of the broadband data was performed using Igor Pro, and a sequential kinetic model convoluted with a Gaussian function held to a FWHM of $200 \mathrm{fs}$, in order to account for the instrument response function. The TAS data collected over the first $10 \mathrm{ps}$ following 334 and $387 \mathrm{~nm}$ excitation was adequately fitted using a three component sequential model (i.e., two lifetimes and an offset), whereas the data following $270 \mathrm{~nm}$ excitation required a four component model (i.e., three lifetimes and an offset). 


\section{Computational methods.}

Static calculations. The topology of the ground and excited potential energy surfaces was explored with the CASSCF method. For specific regions of the $\mathrm{PES}$, the CASPT2 approach, including dynamic correlation (as suggested by previous works) ${ }^{[26]}$, or the consideration of 7 explicit solvent molecules was needed to accurately account for the deactivation mechanism (see supporting information). Final energies were calculated following the MS-CASPT2/SA-CASSCF protocol. An active space of 12 electrons in 9 orbitals (see Figure S1) was used for all the calculations.

Dynamics simulations. A mixed quantum-classical surface-hopping algorithm based on the spin-adiabatic representation has been used. In this scheme the energies, wave functions and couplings were calculated on the fly using a semiempirical $\mathrm{FOMO}-\mathrm{Cl}(8,6)$ scheme including 4 singlet +4 triplet states. The initial conditions were selected using a Boltzmann distribution after an equilibration of the sensitizer within a cluster of 777 water molecules for 100 ps. The swarm of 307 trajectories was propagated until $10 \mathrm{ps}$ with a time step of $0.1 \mathrm{fs}$.

\section{Acknowledgements}

I.C. and L.M.-F. thank the Comunidad Autónoma de Madrid, the Ministerio de Economía y Competitividad (Spain) and the Centro de Computación Científica UAM for a FPU (L.M.-F.) grant, the Projects No. CTQ2012-35513-C02-01 and FOTOCARBON-CM S2013/MIT-2841 and a generous allocation of computational time. G.G. and M.P. acknowledge the financial support of the University of Pisa. M.P. and C.E.C.-H acknowledge funding from the CAREER program of the National Science Foundation (Grants No. CHE-1255084).

Keywords: Sensitizers • DNA damage • singlet oxygen • Timeresolved spectroscopy $\cdot$ Molecular dynamics

[1] a) Nobelprize.org. Nobel Media $A B$ 2014. Web. 24 Feb 2016., <http://www.nobelprize.org/nobel_prizes/medicine/laure ates/1988/press.html> b) Nobelprize.org. Nobel Media $A B$ 2014. Web. $\quad 24 \quad F e b$ 2016., <http://www.nobelprize.org/nobel prizes/medicine/laure ates/1903>.

[2] a) A. Favre, G. Moreno, M. O. Blondel, J. Kliber, F. Vinzens, C. Salet, Biochem. Biophys. Res. Commun. 1986, 141, 847-854; b) C. Salet, M. Bazin, G. Moreno, A. Favre, Photochem. Photobiol. 1985, 41, 617-619.

[3] T. T. Nikiforov, B. A. Connolly, Nucleic Acids Res. 1992 20, 1209-1214.

[4] P. O'Donovan, C. M. Perrett, X. Zhang, B. Montaner, Y.-Z. $\mathrm{Xu}$, C. A. Harwood, J. M. McGregor, S. L. Walker, F. Hanaoka, P. Karran, Science 2005, 309, 1871-1874.

[5] C. Foote, Photochem. Photobiol. 1991, 54, 659

[6] a) A. Massey, Y.-Z. Xu, P. Karran, Curr. Biol. 2001, 11, 1142-1146; b) A. Massey, Y.-Z. Xu, P. Karran, DNA Repair 2002, 1, 275-286. M. Pollum, L. Martínez-Fernández, C. E. CrespoHernández, in Photoinduced Phenomena in Nucleic Acids I: Nucleobases in the Gas Phase and in Solvents, Vol. 355
(Eds.: M. Barbatti, C. A. Borin, S. Ullrich), Springer International Publishing, Cham, 2015, pp. 245-327.

[8] a) O. Reelfs, P. Macpherson, X. Ren, Y.-Z. Xu, P. Karran,

A. R. Young, Nucleic Acids Res. 2011, 39, 9620-9632; b) O. Reelfs, P. Karran, A. R. Young, Photochem. Photobiol. Sci. 2012, 11, 148-154

[9] a) P. Karran, N. Attard, Nat. Rev. Cancer 2008, 8, 24-36;

b) G. B. Elion, Science 1989, 244, 41-47; c) J. E. Murray, J. P. Merrill, J. H. Harrison, R. E. Wilson, G. J. Dammin, New Engl. J. Med. 1963, 268, 1315-1323.

[10] X. Zhang, G. Jeffs, X. Ren, P. O'Donovan, B. Montaner, C. M. Perrett, P. Karran, Y.-Z. Xu, DNA Repair 2007, 6, 344354.

[11] R. Brem, P. Karran, Photochem. Photobiol. 2012, 88, 5-13.

[12] C. Reichardt, C. Guo, C. E. Crespo-Hernández, J. Phys. Chem. B. 2011, 115, 3263-3270.

[13] L. Martinez-Fernandez, L. Gonzalez, I. Corral, Chem. Commun. 2012, 48, 2134-2136.

[14] L. Martinez-Fernandez, I. Corral, G. Granucci, M. Persico, Chem. Sci. 2014, 5, 1336-1347.

[15] a) Y. Harada, C. Okabe, T. Kobayashi, T. Suzuki, T. Ichimura, N. Nishi, Y.-Z. Xu, J. Phys. Chem. Lett. 2010, 1, 480-484; b) C. Reichardt, C. E. Crespo-Hernández, Chem. Commun. 2010, 46, 5963-5965; c) C. Reichardt, C. E. Crespo-Hernández, J. Phys. Chem. Lett. 2010, 1, 22392243; d) M. Pollum, S. Jockusch, C. E. Crespo-Hernández, J. Am. Chem. Soc. 2014, 136, 17930-17933.

[16] a) A. Banyasz, T. Douki, R. Improta, T. Gustavsson, D. Onidas, I. Vayá, M. Perron, D. Markovitsi, J. Am. Chem. Soc. 2012, 134, 14834-14845; b) L. Blancafort, A. Migani, J. Am. Chem. Soc. 2007, 129, 14540-14541; c) W. J. Schreier, P. Gilch, W. Zinth, Annu. Rev. Phys. Chem. 2015, 66, 497-519.

A. Favre, C. Saintomé, J.-L. Fourrey, P. Clivio, P. Laugâa, J. Photochem. Photobiol. B 1998, 42, 109-124.

[18] Y. Harada, T. Suzuki, T. Ichimura, Y.-Z. Xu, J. Phys. Chem. B. 2007, 111, 5518-5524.

[19] The $S_{2 T}$ is not a stationary point of gas phase CASSCF PES. It is only present in highly correlated CASPT2 PES or in CASSCF PES of 4TT hydrogen bonded to water molecules (see supporting information, section 2 and Figure S3).

[20] G. Cui, W. Thiel, J. Phys. Chem. Lett. 2014, 5, 2682-2687.

[21] We caution that although the branching of the $S_{2}$ population predicted by the static calculations could explain the bi-exponential decay of the fluorescence emission observed for 4TT in solution, this putative branching is not supported by the surface hopping dynamics presented in this work (that show no branching), but instead all trajectories follow repeated twisting oscillations that soon or later succeed in causing hops to $S_{1}$ or to the triplet states. Hence, an alternative interpretation is presented in the section where we describe the dynamics simulations. 
[22] M. Kasha, Discuss. Faraday Soc. 1950, 9, 14-19.

[23] M. Pollum, L. A. Ortiz-Rodríguez, S. Jockusch, C. E. Crespo-Hernández, Photochem. Photobiol. 2016, 92, 286292.

[24] a) G. Cui, W.-h. Fang, J. Chem. Phys 2013, 138, 044315; b) J. P. Gobbo, A. C. Borin, Comput. Theor. Chem. 2014, 1040-1041, 195-201; c) J. P. Gobbo, A. C. Borin, J. Phys. Chem. A. 2013, 117, 5589-5596; d) S. Mai, P. Marquetand, L. González, J. Phys. Chem. Lett. 2016, 1978-1983; e) S. Mai, P. Marquetand, L. González, J. Phys. Chem. A. 2015, 119, 9524-9533; f) S. Bai, M. Barbatti, J. Phys. Chem. A 2016, 120, 6342-6350; g) S. Mai, M. Pollum, L. MartínezFernández, N. Dunn, P. Marquetand, I. Corral, C. E. Crespo-Hernández, L. González, Nature Commun. 2016, 7, 13077.

[25] a) C. Reichardt, R. A. Vogt, C. E. Crespo-Hernández, J. Chem. Phys 2009, 131, 224518; b) C. Reichardt, C. Wen, R. A. Vogt, C. E. Crespo-Hernández, Photochem. Photobiol. Sci. 2013, 12, 1341-1350.

[26] J. Segarra-Martí, M. Garavelli, F. Aquilante, J. Chem. Theor. Comput. 2015, 11, 3772-3784. 


\section{FULL PAPER}

Mechanism unraveled! By

combining femtosecond transient absorption spectroscopy and highlevel molecular simulations we have characterized the mechanism by which long-lived triplet excited states in 4-thiothymine are populated. The triplet state is believed to be responsible for the phototoxic properties of 4-

thiothymine following activation by UVA light.

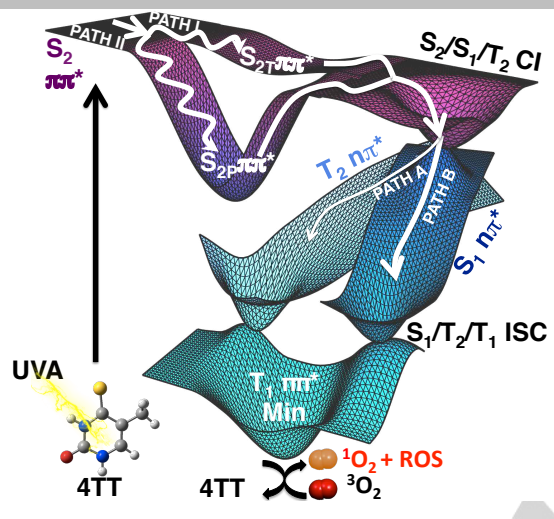

Lara Martínez-Fernandez, Giovanni Granucci*, Marvin Pollum, Carlos E. Crespo-Hernández*, Maurizio Persico, ${ }^{*}$ Inés Corral ${ }^{*}$

Page No. - Page No.

Decoding the molecular basis for the population mechanism of the triplet phototoxic precursors in UVA light-activated pyrimidine anticancer drugs 


\section{Supporting Information}

1. Further Computational Details for the Characterization of the Topology of the PESs

2. Solvent Calculations

3. Comparison of Calculated and Experimental Transient Absorption Spectra

4. Further Results from Molecular Dynamics Simulations

5. Experimental Section 


\section{Further Computational Details for the Characterization of the Topology of the Potential Energy Surfaces.}

The stationary points of the potential energy surface (PES) were optimized at the SACASSCF/ANO-S level of theory and using the minimum number of roots necessary and a $(12,9)$ active space. ${ }^{[1]}$ The ANO-S $\mathrm{S}^{[2]}$ basis set was contracted as $\mathrm{H} 2 \mathrm{~s} 1 \mathrm{p}, \mathrm{C}, \mathrm{N}, \mathrm{O}$ $3 \mathrm{~s} 2 \mathrm{p} 1 \mathrm{~d}$ and $\mathrm{S} 4 \mathrm{~s} 3 \mathrm{p} 2 \mathrm{~d}$. The $(12,9)$ active space includes all the $\pi$ and $\pi^{*}$ orbitals together with the lone pair of the $\mathrm{S}$ atom. All our optimizations exclude the lone from the $\mathrm{O}$ atom (see Figure S1). These stationary points were connected using Minimum Energy Paths (MEPs) calculations, using the intrinsic reaction coordinate (IRC) ${ }^{[3]}$ algorithm (an exemplary MEP can be found in Figure S2). For these MEPs, we used the same protocol as in the optimizations. Final energies at these stationary points of the PES were calculated following the MS-CASPT2//SA-CASSCF/ANO-L (multistate second order perturbation theory on state average complete active space self-consistent field wavefunctions) approach considering 3 singlet and 3 triplet roots, with a $(12,9)$ active space, see Table S1. ${ }^{[4]}$ The ANO-L ${ }^{[5]}$ basis set contraction used was: H 3s $2 \mathrm{p}, \mathrm{C}, \mathrm{N}, \mathrm{O}$ $4 \mathrm{~s} 3 \mathrm{p} 2 \mathrm{~d}$ and $\mathrm{S} 5 \mathrm{~s} 4 \mathrm{p} 2 \mathrm{~d}$. An imaginary level shift of $0.3 \mathrm{au}$ was added to the zero order Hamiltonian to prevent the appearance of intruder states. ${ }^{[6]}$ The IPEA shift was set to zero. ${ }^{[7]}$

Singlet-singlet and Singlet-triplet minimum energy crossing points were optimized at the CASSCF(12,9)/6-31G* level of theory using as starting geometries either those reached from the MEPs or the FC structure.

MS-CASPT2 vertical spectrum, stationary points optimizations, MEPs and final energies calculations were performed with the version 7.8 of MOLCAS program. ${ }^{[8]}$ The optimization of singlet-triplet minimum energy crossing points was performed with MOLPRO 2009. ${ }^{[9]}$ 
Figure S1. CASSCF optimized molecular orbitals included the $(12,9)$ active space used throughout the study. Framed in blue orbitals involved in the $S_{1}$ excitation, in green corresponding to the $S_{2}$ and in purple to the $S_{3}$.
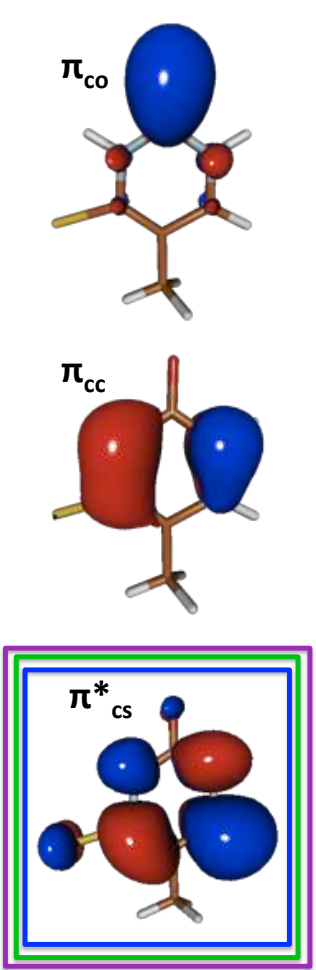
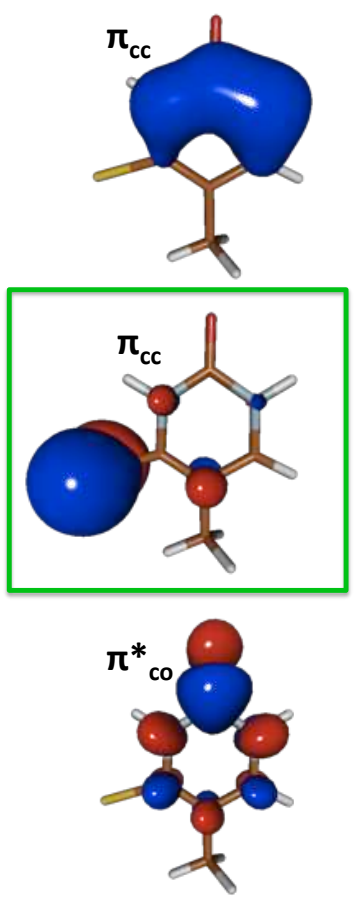
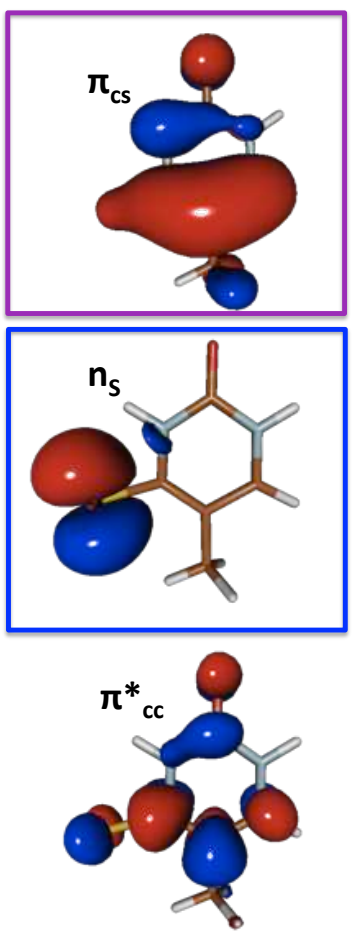

Figure S2. Minimum Energy Paths connecting a) the Franck-Condon region with the $\mathrm{S}_{3} / \mathrm{S}_{2}$ conical intersection region following the $\mathrm{S}_{3}$ excited state gradient $b$ ) the FranckCondon region with the $S_{2 P}$ minimum c) the $S_{2} / S_{1} / T_{2}$ CI region with the $S_{1}\left(n \pi^{*}\right)$ and the $S_{2 P}$ minima $\left.d\right)$ the $S_{2} / S_{1} / T_{2}$ CI region with the $T_{2}\left(n \pi^{*}\right)$ minimum and e) the $S_{1} / T_{2} C I$ region with the $T_{1}\left(\pi \pi^{*}\right)$ minimum

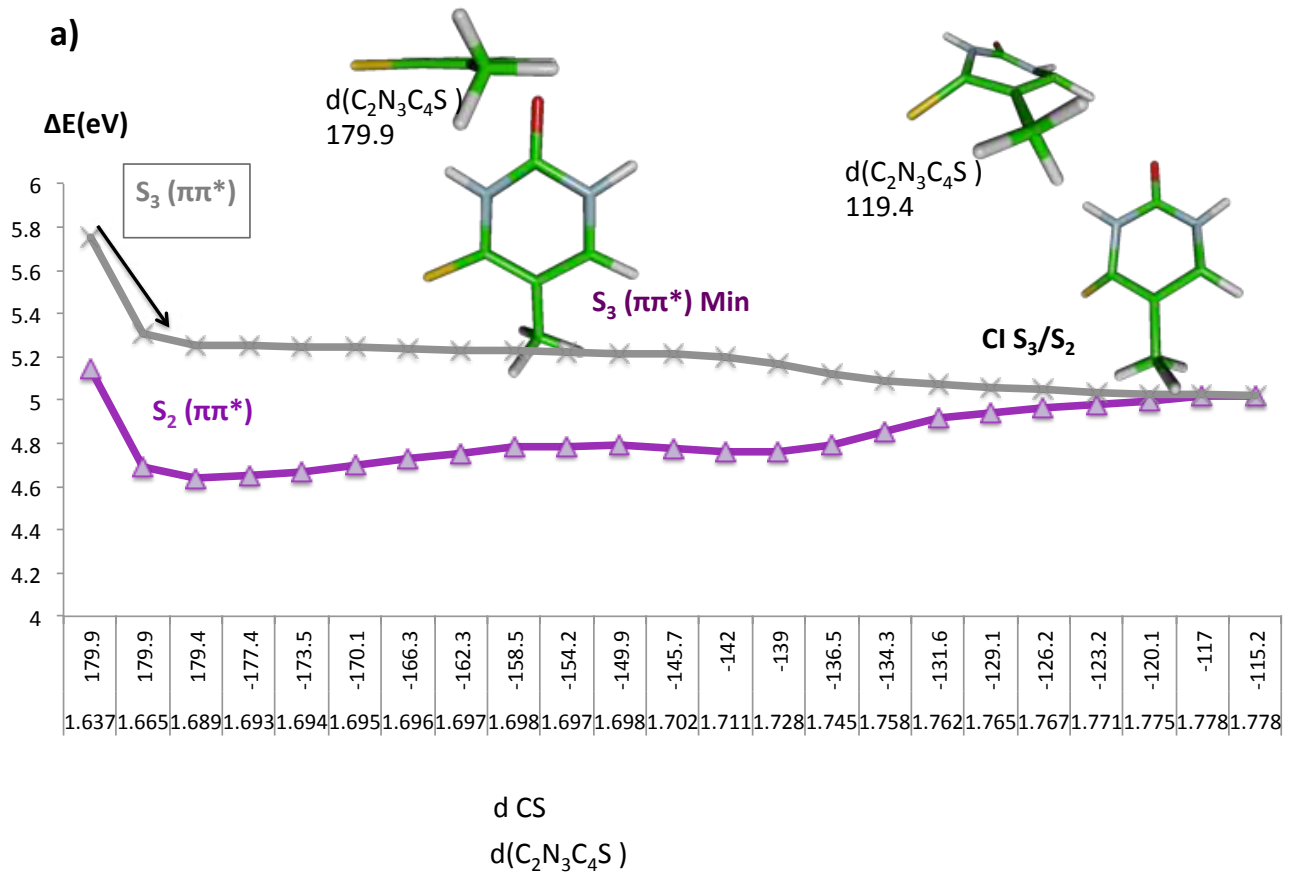


b)

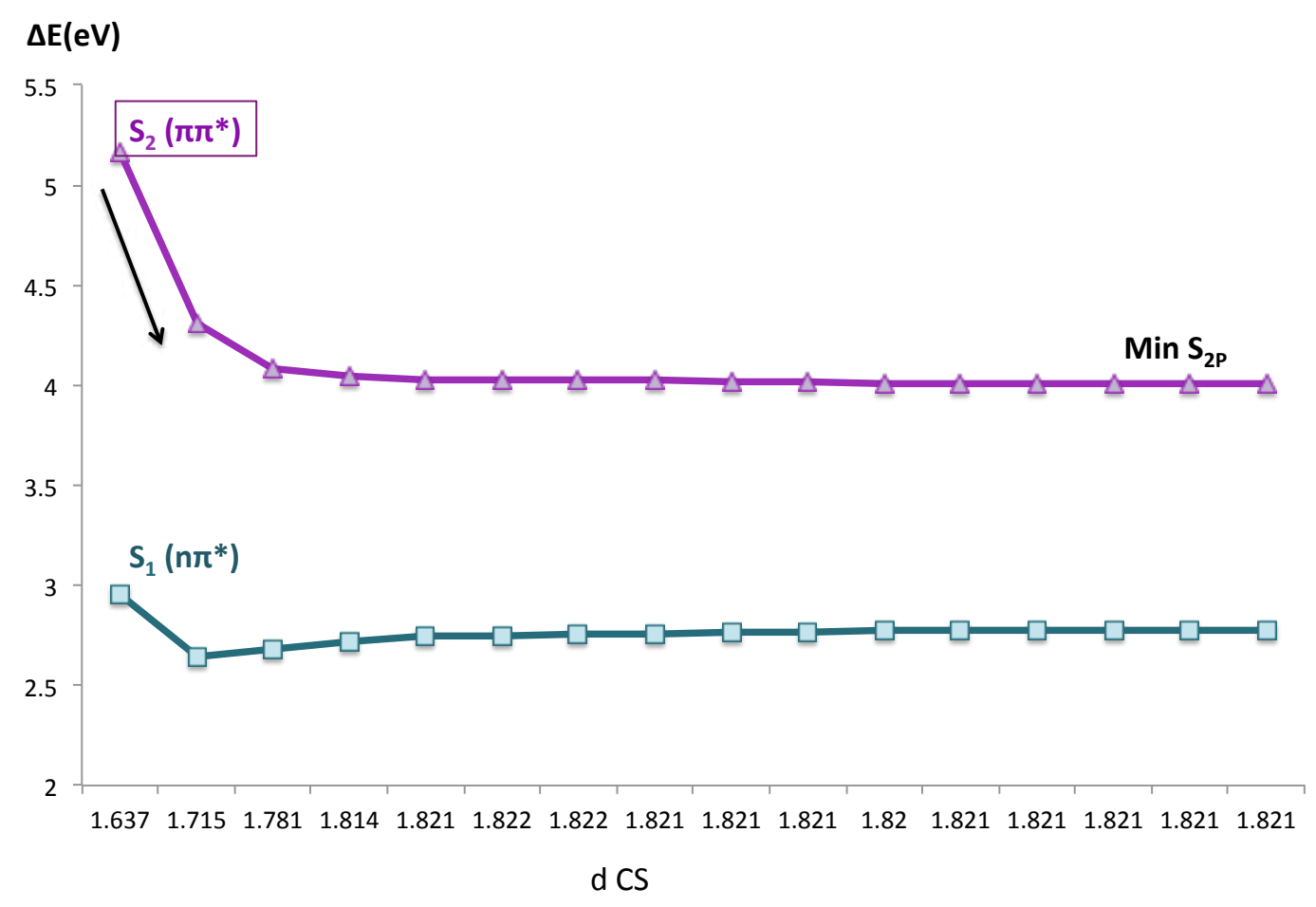

c)

$\Delta \mathrm{E}(\mathrm{eV})$

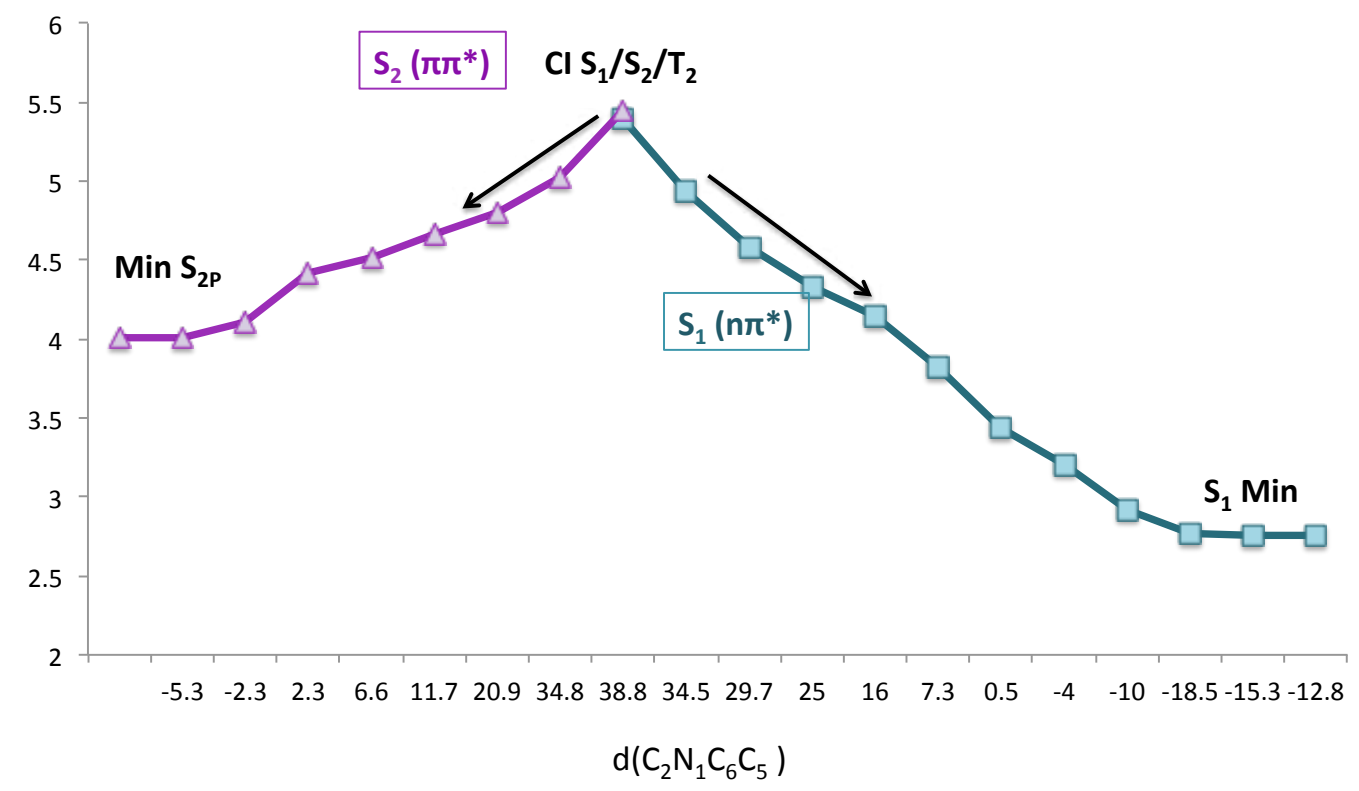


d)

\section{$\Delta \mathrm{E}(\mathrm{eV})$}

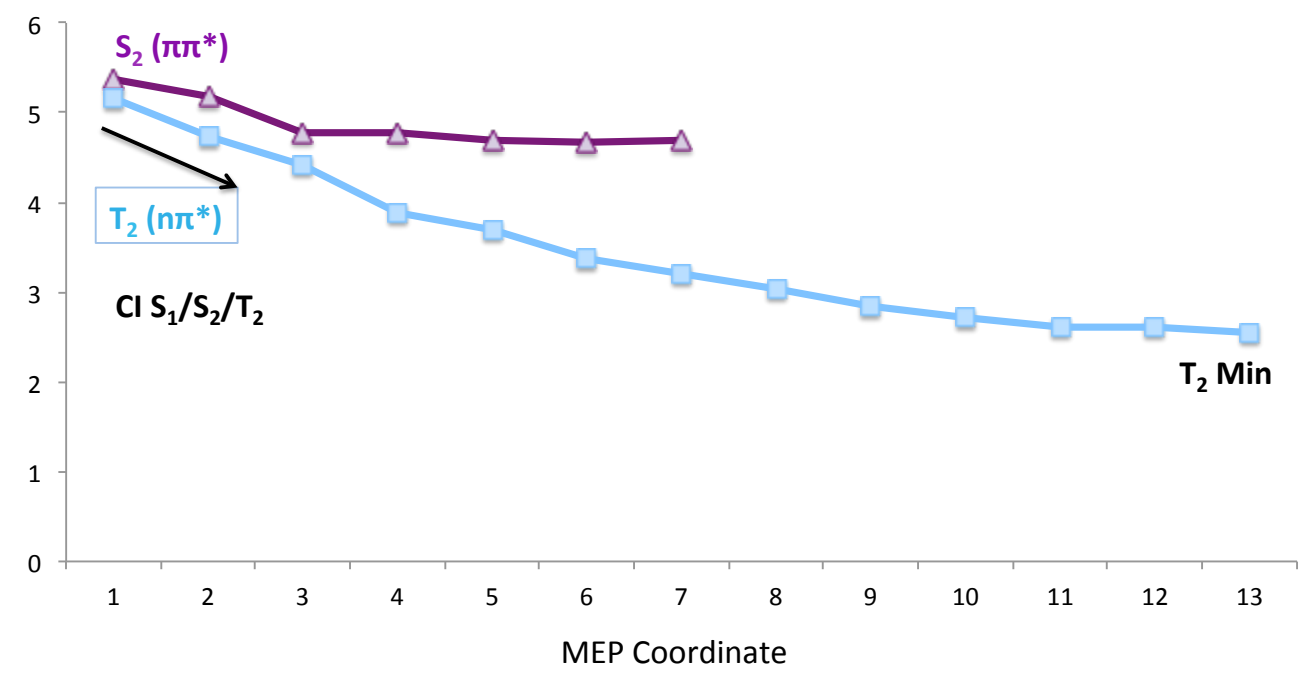

e)

$\Delta \mathrm{E}(\mathrm{eV})$

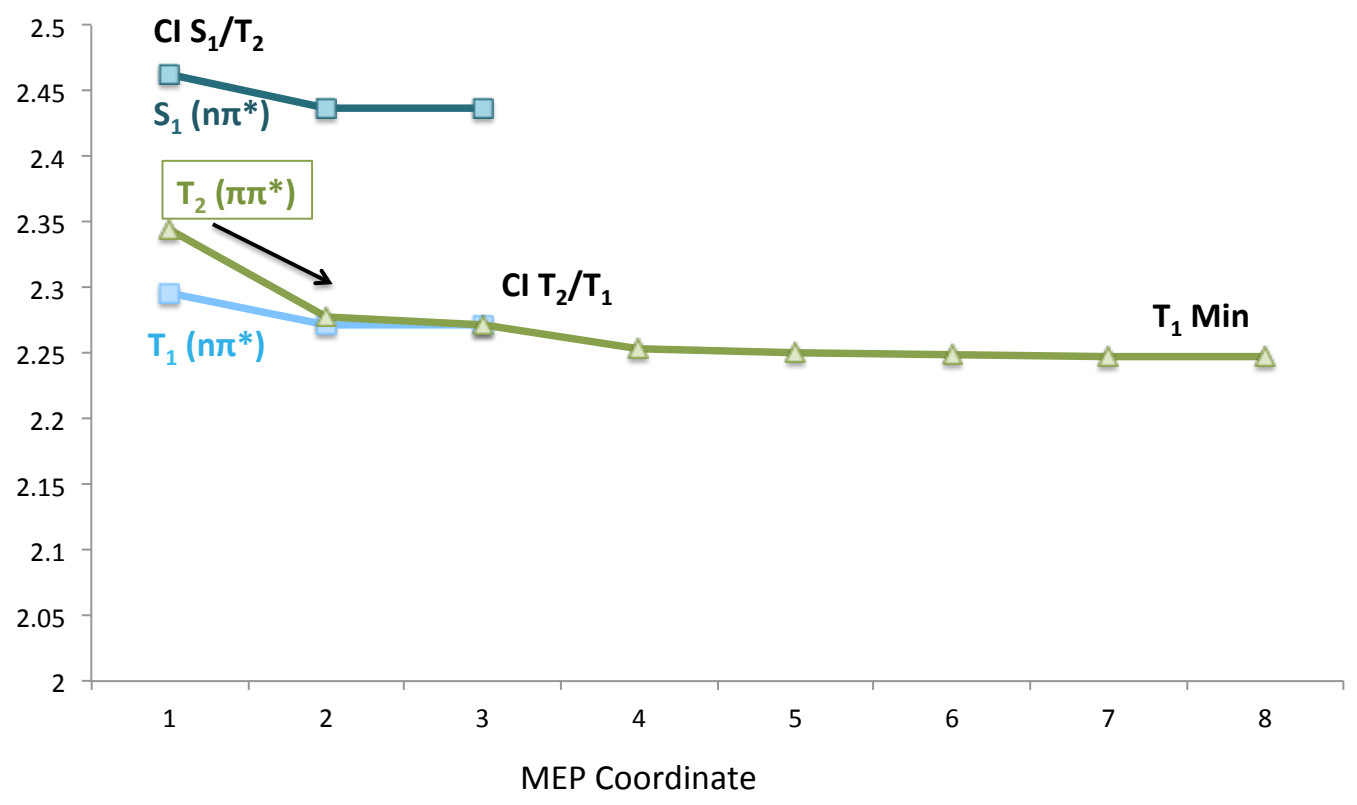


Table S1. MS-CASPT2//CASSCF(12,9)/ANO-L energies (in eV) relative to the ground state for the lowest lying singlet and triplet states at the optimized critical points along the PES of 4-thiothymine.

\begin{tabular}{|c|c|c|c|c|}
\hline Structure & State & $\begin{array}{c}\Delta \mathrm{E} \\
{[\mathrm{eV}]}\end{array}$ & State & $\begin{array}{c}\Delta \mathrm{E} \\
{[\mathrm{eV}]}\end{array}$ \\
\hline FC & $\begin{array}{l}\text { gs } \\
{ }^{1}\left(n \pi^{*}\right) \\
{ }^{1}\left(\pi \pi^{*}\right)\end{array}$ & $\begin{array}{l}0.00 \\
2.87 \\
4.17 \\
\end{array}$ & $\begin{array}{l}3\left(\pi \pi^{*}\right) \\
3\left(n \pi^{*}\right)\end{array}$ & $\begin{array}{l}2.77 \\
2.78 \\
\end{array}$ \\
\hline $\mathrm{S}_{1} \mathrm{n} \pi^{*} \min$ & $\begin{array}{l}\text { gs } \\
1\left(n \pi^{*}\right) \\
1\left(\pi \pi^{*}\right)\end{array}$ & $\begin{array}{l}0.20 \\
2.51 \\
3.57\end{array}$ & $\begin{array}{c}3\left(\pi \pi^{*}\right) \\
3\left(n \pi^{*}\right)\end{array}$ & $\begin{array}{l}2.37 \\
2.47\end{array}$ \\
\hline $\mathrm{S}_{2 \mathrm{~T}} \pi \pi^{*} \min$ & $\begin{array}{l}\text { gs } \\
1\left(n \pi^{*}\right) \\
1\left(\pi \pi^{*}\right)\end{array}$ & $\begin{array}{l}2.81 \\
3.32 \\
4.33\end{array}$ & $\begin{array}{l}{ }^{3}\left(\pi \pi^{*}\right) \\
{ }^{3}\left(n \pi^{*}\right)\end{array}$ & $\begin{array}{l}3.14 \\
3.36\end{array}$ \\
\hline$S_{2 P} \pi \pi^{*} \min$ & $\begin{array}{l}\text { gs } \\
1\left(n \pi^{*}\right) \\
1\left(\pi \pi^{*}\right)\end{array}$ & $\begin{array}{l}0.58 \\
2.73 \\
3.44 \\
\end{array}$ & $\begin{array}{l}3\left(\pi \pi^{*}\right) \\
3\left(n \pi^{*}\right)\end{array}$ & $\begin{array}{l}2.54 \\
2.71\end{array}$ \\
\hline $\mathrm{CI} \mathrm{S}_{2}\left(\pi \pi^{*}\right) / \mathrm{S}_{1}\left(n \pi^{*}\right)$ & $\begin{array}{l}\text { gs } \\
1\left(n \pi^{*}\right) \\
{ }^{1}\left(\pi \pi^{*}\right)\end{array}$ & $\begin{array}{l}3.57 \\
5.05 \\
5.12 \\
\end{array}$ & $\begin{array}{l}3\left(\pi \pi^{*}\right) \\
{ }^{3}\left(n \pi^{*}\right)\end{array}$ & $\begin{array}{l}4.63 \\
5.03\end{array}$ \\
\hline ISC S $2\left(\pi \pi^{*}\right) / T_{2}\left(n \pi^{*}\right)$ & $\begin{array}{l}\text { gs } \\
1\left(n \pi^{*}\right) \\
1\left(\pi \pi^{*}\right)\end{array}$ & $\begin{array}{l}3.29 \\
4.92 \\
5.02 \\
\end{array}$ & $\begin{array}{l}3\left(\pi \pi^{*}\right) \\
3\left(n \pi^{*}\right)\end{array}$ & $\begin{array}{l}4.39 \\
4.91 \\
\end{array}$ \\
\hline$S_{1}\left(n \pi^{*}\right) / T_{2}\left(\pi \pi^{*}\right)$ ISC & $\begin{array}{l}\text { gs } \\
1\left(n \pi^{*}\right) \\
1\left(\pi \pi^{*}\right)\end{array}$ & $\begin{array}{l}0.39 \\
2.56 \\
3.63 \\
\end{array}$ & $\begin{array}{l}3\left(\pi \pi^{*}\right) \\
3\left(n \pi^{*}\right)\end{array}$ & $\begin{array}{l}2.53 \\
2.47 \\
\end{array}$ \\
\hline $\mathrm{CI}_{2}\left(\pi \pi^{*}\right) / \mathrm{T}_{1}\left(\mathrm{n} \pi^{*}\right)$ & $\begin{array}{l}\text { gs } \\
1\left(n \pi^{*}\right) \\
1\left(\pi \pi^{*}\right)\end{array}$ & $\begin{array}{l}0.28 \\
2.53 \\
3.55\end{array}$ & $\begin{array}{l}{ }^{3}\left(\pi \pi^{*}\right) \\
{ }^{3}\left(n \pi^{*}\right)\end{array}$ & $\begin{array}{l}2.41 \\
2.50\end{array}$ \\
\hline $\mathrm{T}_{1} \pi \pi^{*} \min$ & $\begin{array}{l}\text { gs } \\
1\left(n \pi^{*}\right) \\
{ }^{1}\left(\pi \pi^{*}\right)\end{array}$ & $\begin{array}{l}0.20 \\
2.54 \\
3.58\end{array}$ & $\begin{array}{l}{ }^{3}\left(\pi \pi^{*}\right) \\
{ }^{3}\left(n \pi^{*}\right)\end{array}$ & $\begin{array}{l}2.34 \\
2.49\end{array}$ \\
\hline $\mathrm{CI} \mathrm{S}_{1}\left(\mathrm{n} \pi^{*}\right) / \mathrm{S}_{0}$ & $\begin{array}{l}\text { gs } \\
1\left(n \pi^{*}\right) \\
1\left(\pi \pi^{*}\right)\end{array}$ & $\begin{array}{l}4.43 \\
4.54 \\
6.34\end{array}$ & $\begin{array}{l}{ }^{3}\left(\pi \pi^{*}\right) \\
{ }^{3}\left(n \pi^{*}\right)\end{array}$ & $\begin{array}{l}4.47 \\
4.93 \\
\end{array}$ \\
\hline $\mathrm{ISC}_{1}\left(\pi \pi^{*}\right) / \mathrm{S}_{0}$ & $\begin{array}{l}\text { gs } \\
1\left(n \pi^{*}\right) \\
1\left(\pi \pi^{*}\right)\end{array}$ & $\begin{array}{l}4.42 \\
5.02 \\
5.62 \\
\end{array}$ & $\begin{array}{l}3\left(\pi \pi^{*}\right) \\
{ }^{3}\left(n \pi^{*}\right)\end{array}$ & $\begin{array}{l}4.44 \\
5.06 \\
\end{array}$ \\
\hline
\end{tabular}




\section{Solvation Models \\ 2.1 QM calculations: Effect of explicit QM water molecules in the Potential Energy Surfaces.}

The poor comparison between the excited state dynamics of 4-thiothymine (4TT) captured by femtosecond spectroscopic experiments and the main deactivation mechanisms based on the analysis of the gas phase potential energy surface motivated the study of the influence of explicit solvent molecules (water) in the shape of the PES.

In fact, the first stages of the decay mechanism $\left(\mathrm{S}_{2} / \mathrm{S}_{1}\right.$ internal conversion) were detected to be especially sensitive to solvent interactions. Gas phase CASSCF calculations predict a unique minimum in the $S_{2}$ potential, $S_{2 \mathrm{P}}$, characterized by a planar geometry. This minimum is separated from the $S_{2} / S_{1}$ internal conversion funnel by an energy barrier that amounts to $1.6 \mathrm{eV}$ (see Figure S3). This scenario is not compatible with a rise time of the triplets in the femtosecond time scale.

Only after incorporating dynamic correlation via CASPT2 approach or by explicitly considering a minimum number of water molecules directly interacting with the chromophore, it is possible to locate a second minimum, $S_{2 \mathrm{~T}}$, structurally similar to the $\mathrm{CIS}_{2} / \mathrm{S}_{1}$. Moreover, this reduced number of water molecules surrounding the thiated nucleobase also produces a remarkable stabilization of the $\mathrm{CIS}_{2} / \mathrm{S}_{1}$ which decreases its energy by $\sim 0.4 \mathrm{eV}$ (see Figure S3). At contrast, the effect of water solvent on the $\mathrm{S}_{1}$ minimum was found to be negligible, in line with the results reported in ref [10]. Thus, the explicit incorporation of chromophore-water interactions to our model simulations allows unveiling a second more favorable path for the decay of 4TT, that requires surmounting a low energy barrier of $0.1 \mathrm{eV}$, which would compete with a slow deactivation pathway, along which an energy barrier of at least $1 \mathrm{eV}$ needs to be overcome.

In order to determine the minimum number of water molecules accounting for a topology of the $S_{2}$ potential energy surface consistent with the experimental dynamics, we considered a $100 \mathrm{ps} \mathrm{QM} / \mathrm{MM}$ equilibration dynamics simulation for a cluster consisting of the sensitizer plus a bulk of 777 water molecules (Figure S3b). These simulations revealed 7 water molecules strongly interacting with the sensitizer and, thus, defining the first solvation shell, as depicted in Figure S3a. This cluster consisting of the sensitizer $\bullet 7 \mathrm{H}_{2} \mathrm{O}$ was used for the characterization of the stationary and critical points involving the $\mathrm{S}_{2}$ potential. 
Figure S3. a) MS-CASPT2//CASSCF energies (in black) for the first stages of the deactivation mechanism of 4TT considering the 7 water molecules cluster in the inset. In red, the MS-CASPT2//CASPT2 energies for the same critical points considering the isolated chromophore. b) 4TT within the bulk of water molecules used during the QM/MM dynamics simulations

a)

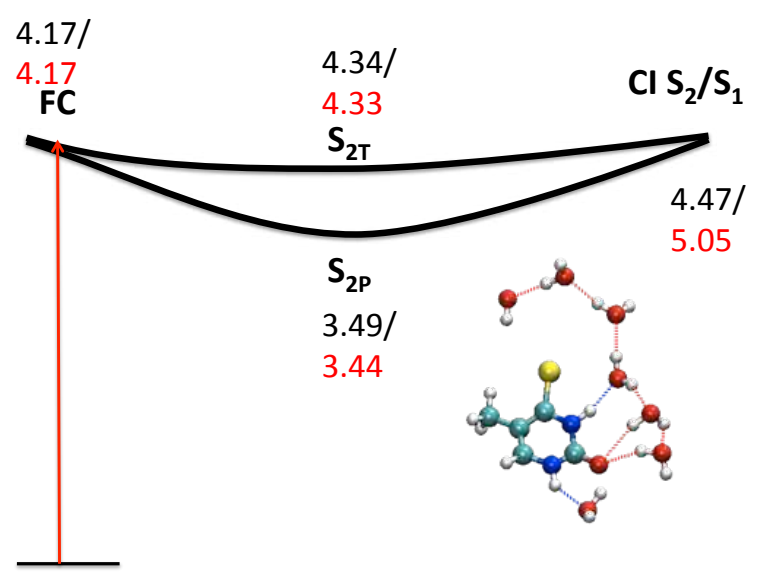

b)

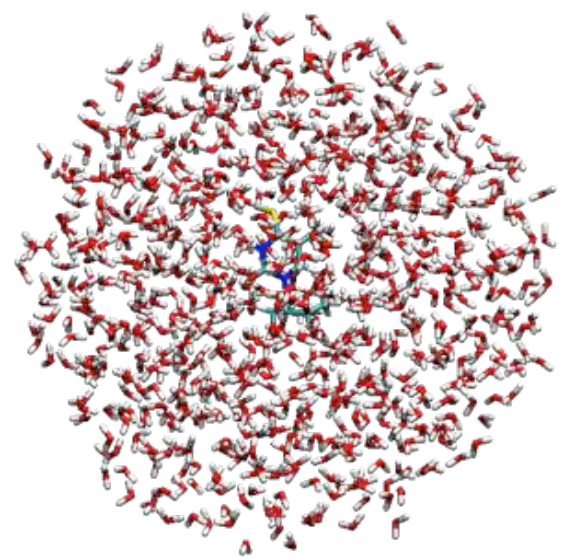

2.2 Solvation Model for the QM/MM dynamics simulations

From these results, we conclude that accounting for solvent effects seems to be determinant to model the decay of 4TT. Thus, molecular dynamics simulations were then performed following a QM/MM approach.

In the QM/MM excited state dynamics simulations, 4TT has been inserted in a large cluster of 777 water molecules (see Figure 3b), represented using the TIP3P MM potential. The MM part comprised also the sugar of 4-thiothymidine, which has been represented using the OPLS force field. In the QM/MM simulations the electrostatic embedding has been adopted and the covalent QM-MM link has been described using the "connection atom" approach of Antes and Thiel. ${ }^{[1]}$ A constraining harmonic potential has been added at the boundary of the solvent sphere to prevent any evaporation of water molecules from the cluster during the dynamics simulations. In evaluating the interaction with the QM part, the standard OPLS and TIP3P charges are scaled by the factor 0.82 .

\subsection{Solvation Model for the energy and electron transfer processes}

The thermodynamic analysis of the energy and electron transfer processes from the 4TT and 6-TG triplet minima towards ground state oxygen molecules was performed at the CASPT2 level of theory considering the effect of water as solvent using the polarizable continuum model (PCM) implicit model, as implemented in MOLCAS 8 package. 


\section{Comparison of experimental and theoretically predicted transient absorption spectra.}

In order to confirm the decay mechanism inferred from static and dynamics simulations, we have theoretically modeled transient absorption spectra (TAS) at particular delay times and compared these results with the experimentally registered analogues. The ideal simulation of the time resolved transient experimental spectra would involve computing vertical absorptions based on the excited state dynamics. This, however, would imply calculating hundreds (as many as the number of trajectories considered) of vertical spectra at each time step, being very time consuming and impractical. As a suitable and more computationally affordable approximation, we compute the absorptions at the position of the excited singlet and triplet minima, which the wave packet is expected to visit for longer times.

These calculations were performed at MS-CASPT2/CASSCF/ANO-L level of theory considering 25 singlet or triplet roots at each possible excited state intermediate. These intermediates considered were: the $S_{2}\left(\pi \pi^{*}\right)$ excited state at the position of the planar and twisted minima $\left(S_{2 \mathrm{P}}\right.$ and $\left.\mathrm{S}_{2 \mathrm{~T}}\right)$, the $n \pi^{*}$ excited state at the position of its minimum $\left(\mathrm{S}_{1}\right)$ and the absorptions of the triplets $\mathrm{T}_{1}\left(\pi \pi^{*}\right)$ and $\mathrm{T}_{2}\left(\mathrm{n} \pi^{*}\right)$ at their corresponding triplet minima $\left(\mathrm{T}_{1}\right.$ and $\mathrm{T}_{2}$ ). In the case of the short 270 wavelength, we have also considered the transient spectra of the $S_{3}\left(\pi \pi^{*}\right)$ excited state computed at its optimized minimum. Stimulated emission was not simulated in this paper.

Then, these vertically absorption spectra at the excited intermediates were subsequently combined at different extents to best match the experimental recorded TAS. By doing so, we can roughly estimate which is the population lying at each states for each particular delay time, providing an alternative procedure to figure out the kinetic decay mechanism of 4TT. This procedure has been applied to the three different experiments exciting at 387, 334 and $270 \mathrm{~nm}$ and the results are presented in Figure S4-S6 and discussed in the main paper. 
Figure S4. Experimental (upper panel) and calculated (lower panel) Transient Absorption Spectra at representative lifetimes following $387 \mathrm{~nm}$ excitation.

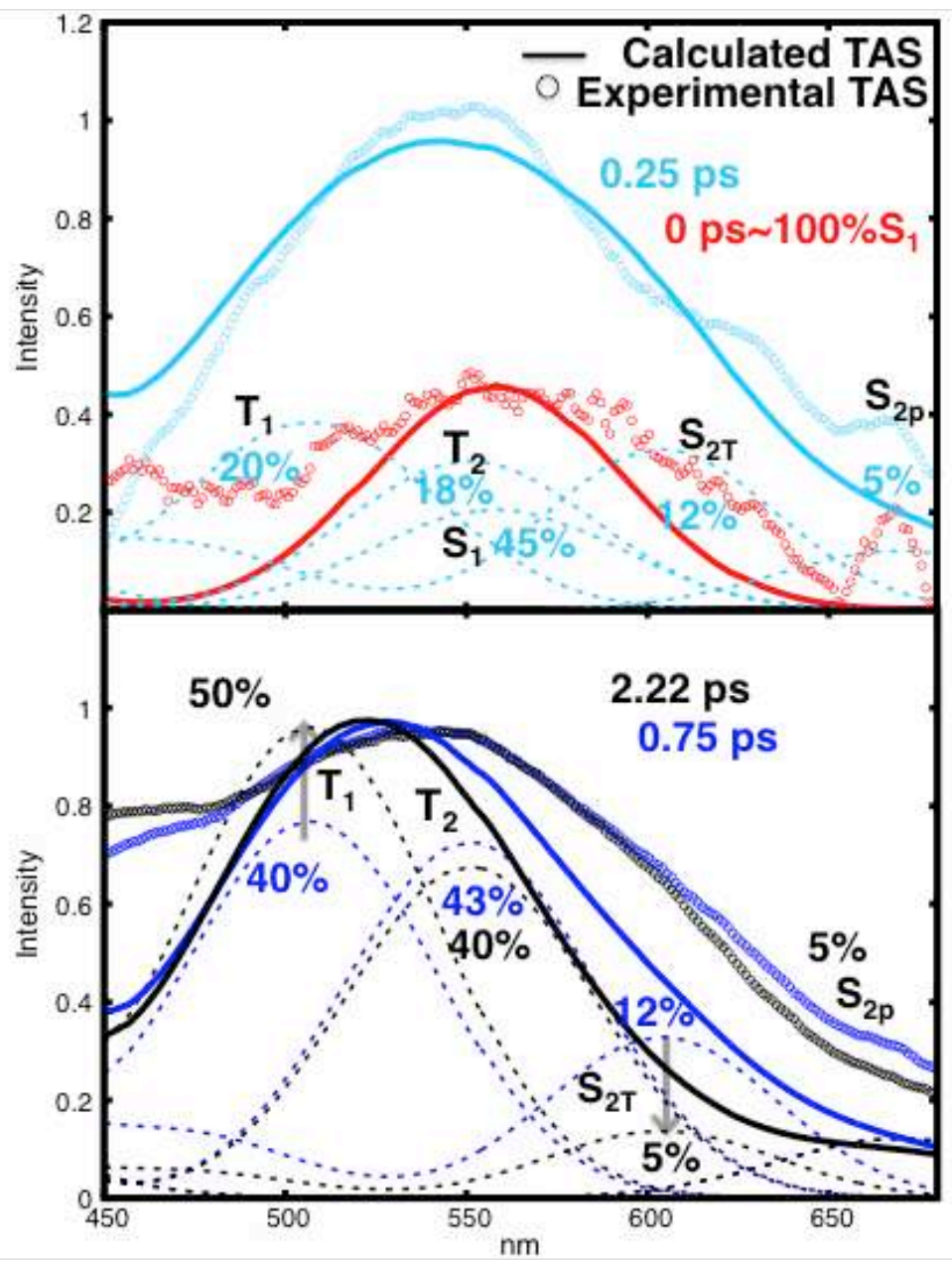


Figure S5. Experimental (upper panel) and calculated (lower panel) Transient Absorption Spectra at representative lifetimes following $334 \mathrm{~nm}$ excitation.

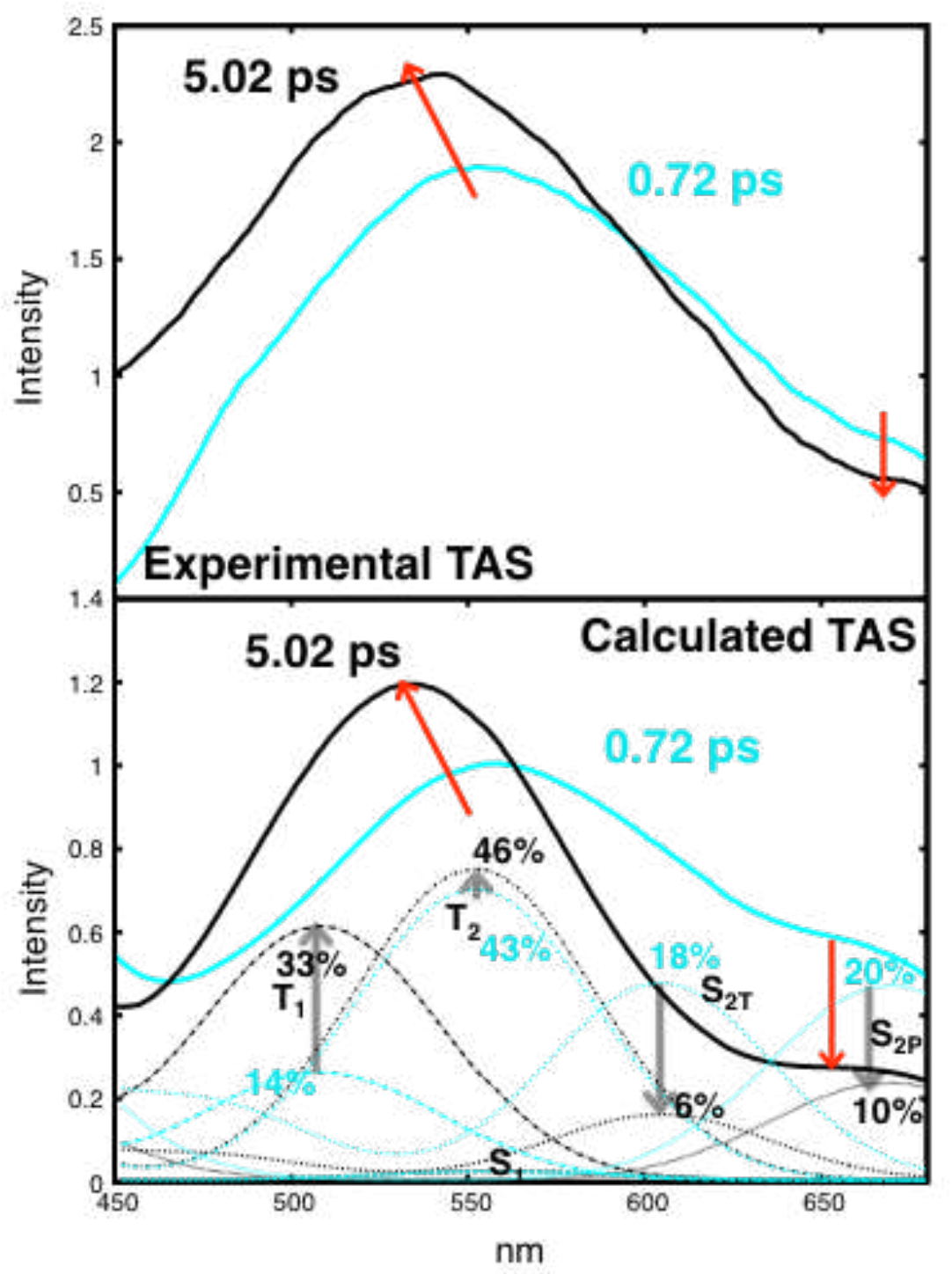


Figure S6. Experimental (upper panel) and calculated (lower panel) Transient Absorption Spectra at representative lifetimes following $270 \mathrm{~nm}$ excitation.

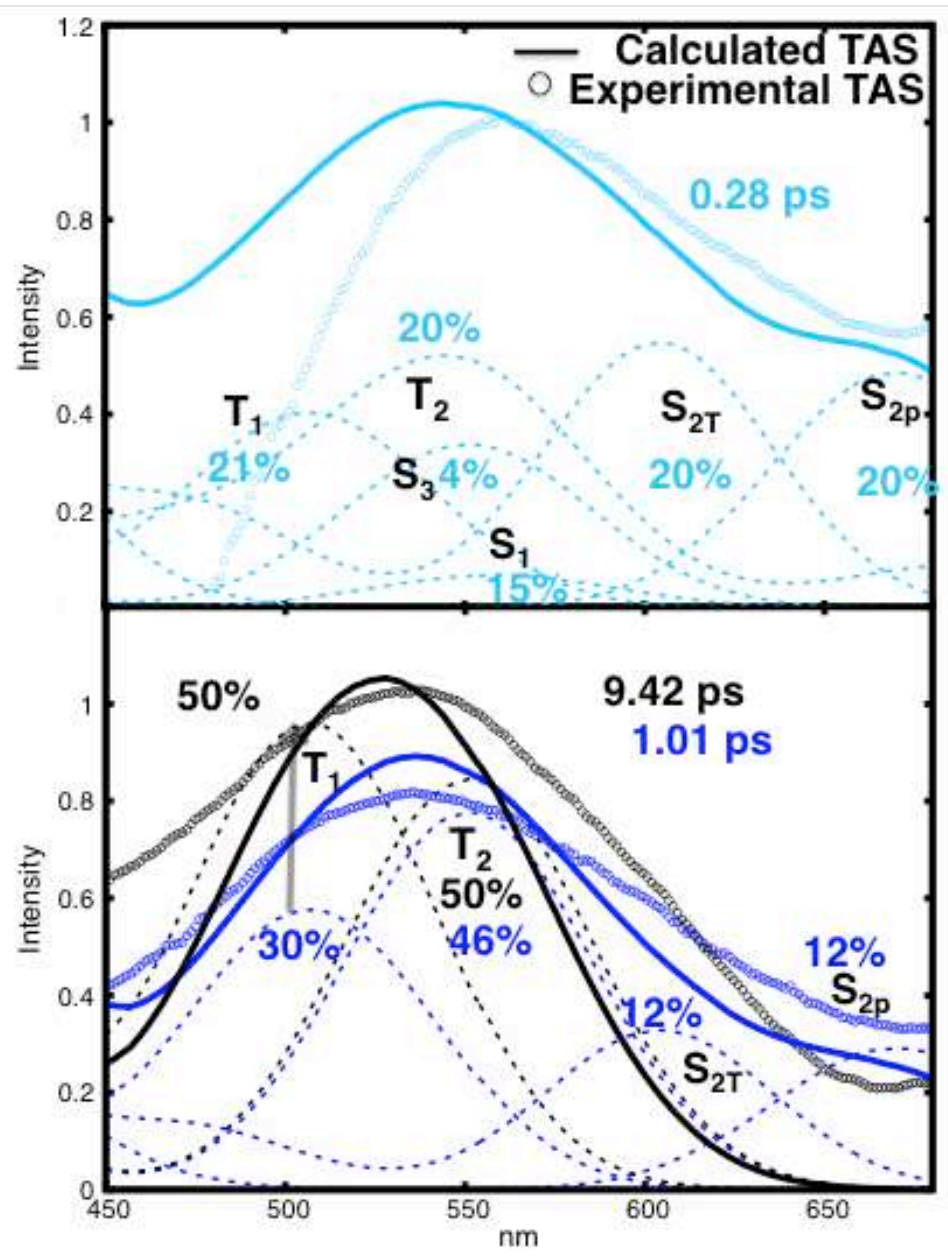




\section{Further results from the $\mathrm{QM} / \mathrm{MM}$ excited state dynamics simulations.}

Figure S7. Decay of the $\mathrm{S}_{2}$ population (blue) together with the twisting of the $\mathrm{N}_{1}-\mathrm{C}_{6}-\mathrm{C}_{5}$ $\mathrm{C}_{7}$ dihedral angle (green) and this dihedral corrected with the standard deviation (red).

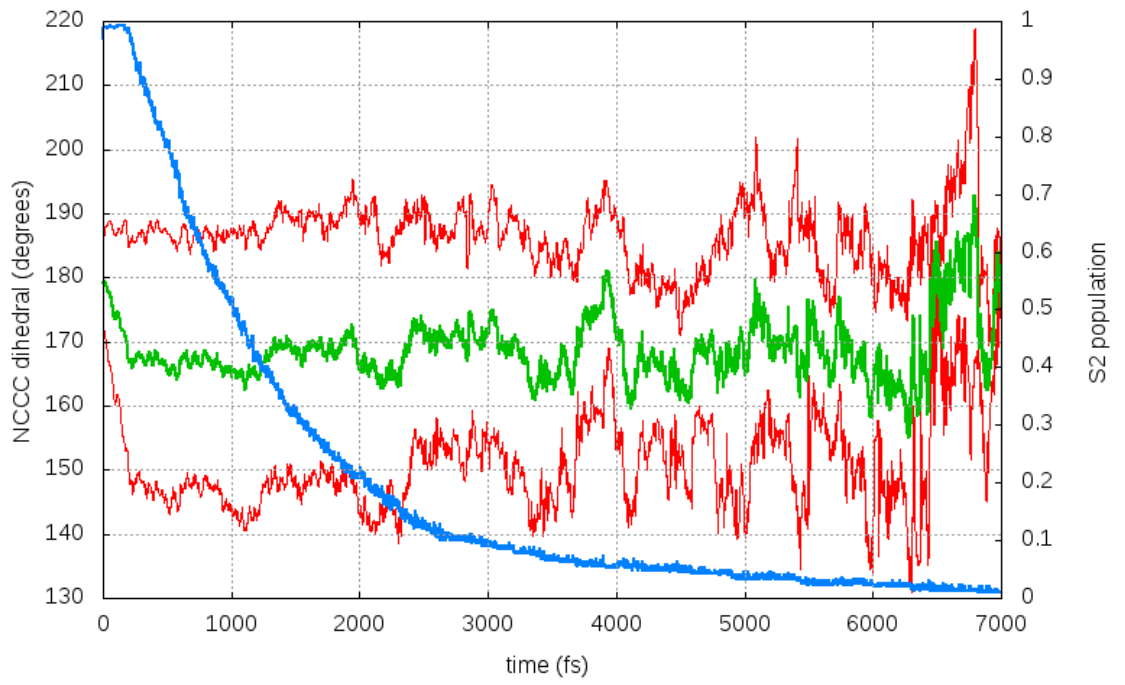

Figure S8. Distribution of the number of trajectories in the $S_{2}$ potential as a function of the $\mathrm{N}_{1}-\mathrm{C}_{6}-\mathrm{C}_{5}-\mathrm{C}_{7}$ dihedral angle. Different colors indicate different times from 0 (purple) to 1 (red) ps.

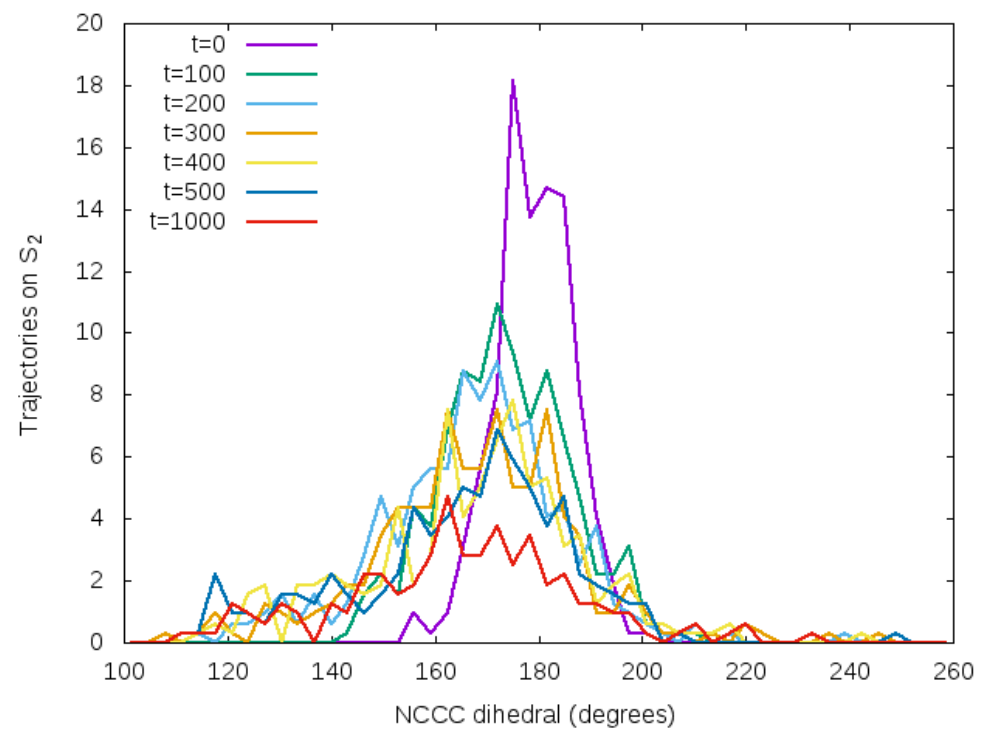




\section{Experimental Section}

Figure S9. Representative kinetic traces taken at a probe wavelength of $650 \mathrm{~nm}$ for 4TT following $334 \mathrm{~nm}$ excitation in the indicated solvent.

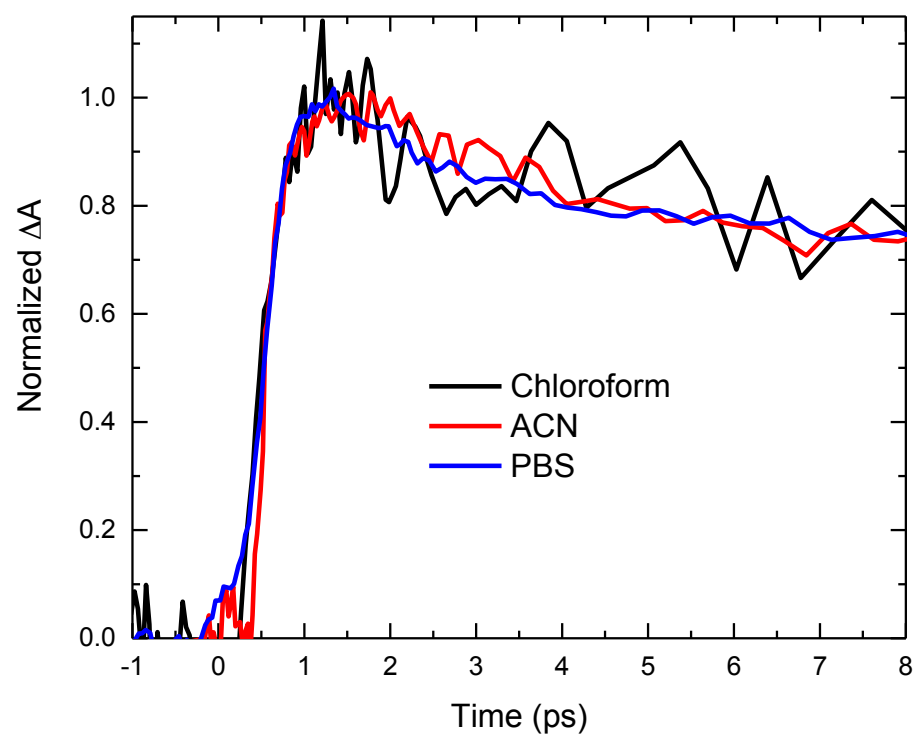

Figure S10. Kinetic traces taken at representative probe wavelengths for 4TT in PBS (a) and ACN (b) following $334 \mathrm{~nm}$ excitation. Fit lines are from a global analysis of broadband transient absorption data and produced the lifetimes reported in Table 2 .
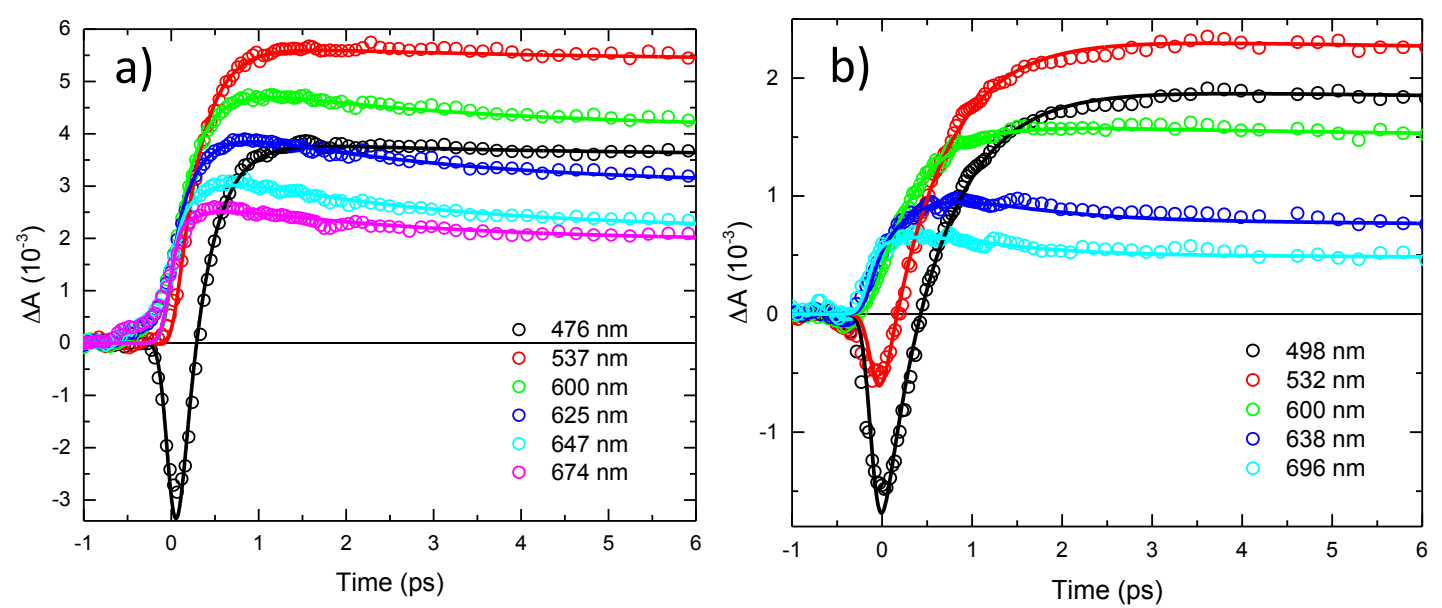
Figure S11. Kinetic traces taken at representative probe wavelengths for 4TT in ACN following $387 \mathrm{~nm}$ excitation (a) and in PBS (b) and ACN (c) following $270 \mathrm{~nm}$ excitation. Fit lines are from a global analysis of broadband transient absorption data and produced the lifetimes reported in Table 2.
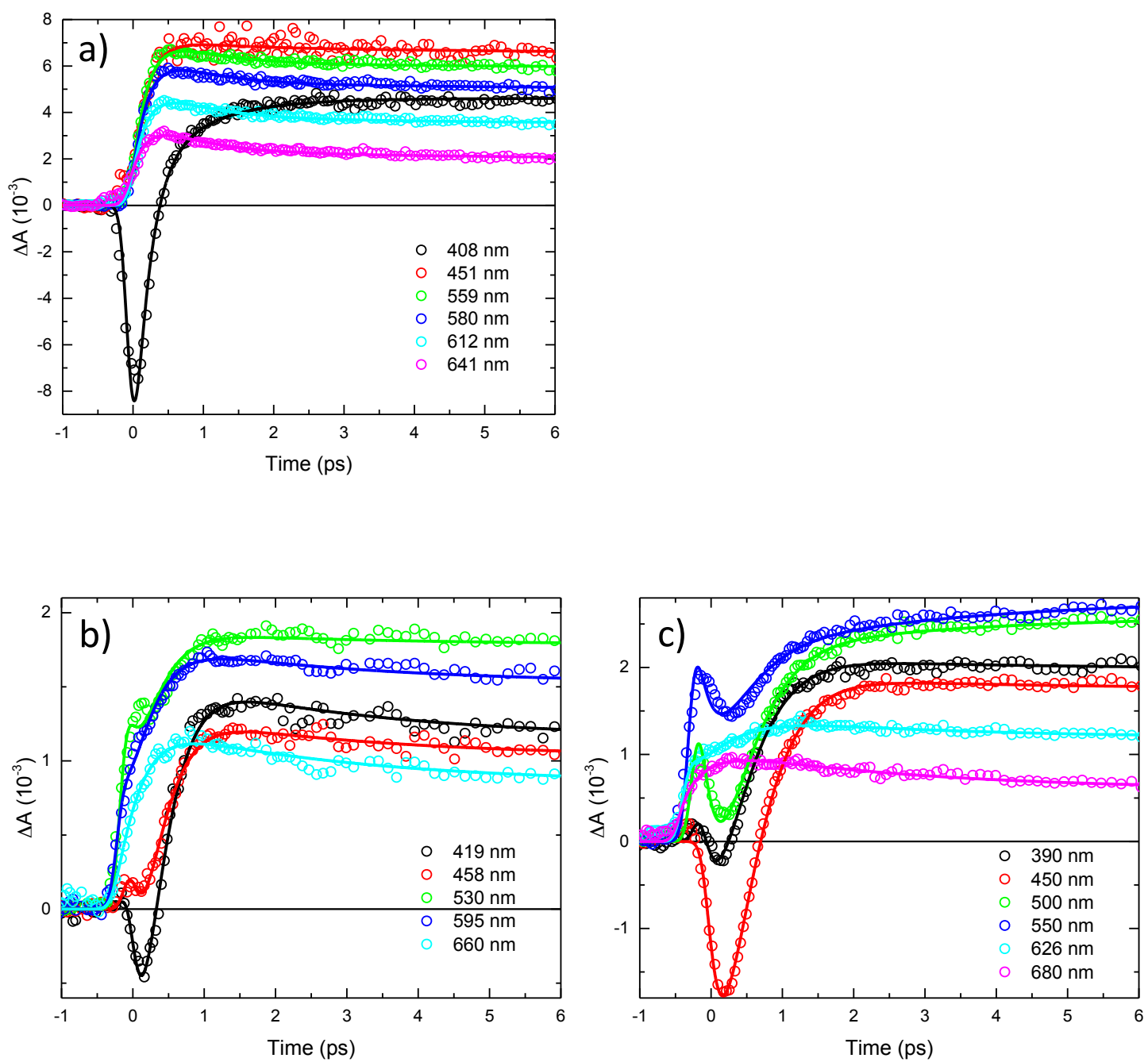


\section{References}

[1] B. O. Roos, In Ab initio Methods in Quantum Chemistry II Lawley, K. P.Ed.; Wiley: Chichester 1987.

[2] K. Pierloot, B. Dumez, P. O. Widmark, B. O. Roos, Theor. Chim. Acta 1995, 90, 87.

[3] A. Migani, M. Olivucci, in Conical Intersections: Electronic Structure, Dynamics and Spectroscopy, Vol. 15 (Eds.: W.

Domcke, D. Yarkony, H. Köppel), World Scientific, 2004.

[4] a) K. Andersson, P. Å. Malmqvist, B. O. Roos, J. Chem. Phys. 1992, 96, 1218;

b) J. Finley, P. Å. Malmqvist, B. O. Roos, L. Serrano-Andrés, Chem. Phys. Lett. 1998, 288, 299.

[5] P. O. Widmark, P. Å. Malmqvist, B. O. Roos, Theor. Chim. Acta 1990, 77, 291.

[6] N. Forsberg, P. Å. Malmqvist, Chem. Phys. Lett. 1997, 274, 196.

[7] G. Ghigo, B. O. Roos, P. A. Malmqvist, Chem. Phys. Lett. 2004, 396, 142.

[8] F. Aquilante, L. De Vico, N. Ferré, G. Ghigo, P. Å. Malmqvist, P. Neogrády, T. B. Pedersen, M. Pitoňák, M. Reiher, B. O. Roos, L. Serrano-Andrés, M. Urban, V. Veryazov, R. Lindh, J. Comput. Chem. 2010, 31, 224.

[9] H.-J. Werner, P. J. Knowles, R. Lindh, F. R. Manby, M. Schutz, P. Celani, T. Korona, G. Rauhut, R. D. Amos, A. Bernhardsson, A. Berning, D. L. Cooper, M. J. O. Deegan, A. J. Dobbyn, F. Eckert, C. Hampel, G. Hetzer, A. W. Lloyd, S. J. McNicholas, W. Meyer, M. E. Mura, A. Nicklass, P. Palmieri, U. Schumann, H. Stoll, A. J. Stone, R. Tarroni, T. Thosteinsson, v. MOLPRO, a package of ab initio programs.

[10] S. Bai, M. Barbatti, J. Phys. Chem. A 2016, 120, 6342-6350.

[11] a) I. Antes, W. Thiel, J. Phys. Chem. A 1999, 103, 9290-9295; b) A. Toniolo, C. Ciminelli, G. Granucci, T. Laino, M. Persico, Theor. Chem. Acc. 2004, 93, 270 279. 